\title{
Particle imaging techniques for volumetric three-component (3D3C) velocity measurements in microfluidics
}

Received: 26 May 2011/Accepted: 31 August 2011/Published online: 8 October 2011

(C) The Visualization Society of Japan 2011

\begin{abstract}
The reliable measurement of the 3D3C velocity field in microfluidic devices becomes more and more important for future optimization and developments for lab-on-a-chip applications or point-of-care medical diagnosis systems. In the past years, different particle-based imaging methods, such as confocal scanning microscopy, holography, stereoscopic and tomographic imaging or approaches based on defocused particle images or optical aberrations have been developed and applied successfully to measure velocity fields in microfluidic systems. The benefits and drawbacks of these methods will be discussed in detail as the proper understanding of the measurement principle is essential to select the most appropriate technique for a desired measurement application. Once an imaging method is chosen, the velocity can be estimated by correlation-based methods or tracking approaches. The advantages and disadvantages of both methods and the importance of image preprocessing will also be discussed in detail.
\end{abstract}

Keywords Microfluidics · Volumetric velocity measurements · PIV · PTV ·

Confocal scanning microscopy $\cdot$ Holography $\cdot$ Astigmatism $\cdot$ Spatial resolution $\cdot$ Sum of correlation

\section{Introduction}

\subsection{Introduction to $\mu \mathrm{PIV}$}

In the past years, more and more complex microfluidic devices such as micro-mixers, micro-bioreactors and micro-heat exchangers, among others, have been designed for life science and process engineering applications (Yoshida 2005). Although the Reynolds numbers for microfluidic devices are often fairly low $R e \leq 100$ and the state of the flow is laminar, numerical flow simulations are often difficult or even impossible to perform. Difficulties arise due to complex fluid properties and flow geometries, surface phenomena such as electrokinetic or electrophoretic forces, electric or magnetic field gradients, fluidstructure interaction, etc. A comprehensive review about the manifold of physical effects on these small scales can be found in Squires and Quake (2005) and the references herein. However, for a proper and effective design of the microfluidic device and the validation of numerical flow simulations, the reliable experimental characterization of three-dimensional flows is an important issue.

Therefore, the $\mu \mathrm{PIV}$ (particle image velocimetry) method was introduced for the measurement of 2D2C (two-dimensional, two-components) velocity fields in microfluidic devices. Today, $\mu \mathrm{PIV}$ has become one of

C. Cierpka $(\bowtie) \cdot$ C. J. Kähler

Institute for Fluid Mechanics and Aerodynamics, Bundeswehr University Munich,

85577 Neubiberg, Germany

E-mail: Christian.Cierpka@unibw.de

C. J. Kähler

E-mail: Christian.Kaehler@unibw.de 
the most widespread techniques to measure the fluid velocities in microfluidic devices. The main steps include the imaging of tiny fluorescent particles, illuminated twice in a microchannel, by epi-fluorescence microscopy (and referred hereafter as standard microscopy), the evaluation of the particle image displacement with digital image processing algorithms and the conversion of the particle image displacement into physical space by a proper calibration. The adaptation of macroscopic PIV using a microscope was first described by Santiago et al. (1998) and successfully applied to microchannel flows (Meinhart et al. 1999; Koutsiaris et al. 1999; Sugii and Okamoto 2003). Most of the 3D techniques share the same equipment and rely on similar basic principles, schematically shown on the left side of Fig. 1. In standard $\mu$ PIV, the transparent flow of interest is usually seeded with fluorescent tracer particles, imaged by high-quality microscope objective lenses. The whole microfluidic channel is then illuminated by a laser, or another bright light source (continuous or pulsed) such as high-power LEDs (Hagsäter et al. 2008). To homogenize the beam profile, a diffuser plate is often used. If a white light source is deployed, a filter cube is applied to only let short-wavelength (green) light through. This short-wavelength light is then absorbed by the tracer particles' fluorescent dye and a distribution of longer wavelength, red light is emitted. A filter is used to only let the emitted red light pass onto the camera. The major benefit of the fluorescence is that reflections from the channel walls can be removed from the images at the cost of weaker fluorescence signals. The particle images are then recorded by a digital CCD (Charge Coupled Device), CMOS (Complementary Metal Oxide Semiconductor) or intensified camera. For the benefits of either, the interested reader is referred to Hain et al. (2007). The synchronization of the whole system is assured by the synchronizing electronics. Applying a proper calibration from image space to the real physical coordinates, the recorded images can be used to estimate the velocity of the fluid by measuring the displacement of the tracer particles, using correlation or particle tracking algorithms. The major difficulty arises from the fact that in contrast to standard PIV, where the measurement plane is defined by a laser light sheet, the measurement plane in $\mu \mathrm{PIV}$ is determined by the depth of focus of the optical system, which can reach several micrometers (Olsen and Adrian 2000; Bourdon et al. 2004; Rossi et al. 2011b; Kloosterman et al. 2010). Out-of-focus particles, as shown on the right side of Fig. 1, contribute to the cross correlation and hence add a bias to the measurement in case of out-of-plane gradients (depth of correlation). A first model to derive a measure for the depth of correlation (DOC), which limits the depth resolution was proposed by Olsen and Adrian (2000). Without going into detail on the assumptions for their model, they came up with the following equation:

$$
\mathrm{DOC}=2 z_{\text {corr }}=2\left\{\frac{(1-\sqrt{\varepsilon})}{\sqrt{\varepsilon}}\left[f^{\# 2} d_{\mathrm{p}}^{2}+\frac{5.95(M+1)^{2} \lambda^{2} f^{\# 4}}{M^{2}}\right]\right\}^{1 / 2}
$$

where $f^{\#}$ is the focal number of the lens, $d_{\mathrm{p}}$ is the particle diameter, $\lambda$ is the wavelength of the light emitted by the fluorescent particles and $M$ is the magnification of the system. The parameter $\varepsilon$ is usually set to 0.01 ,

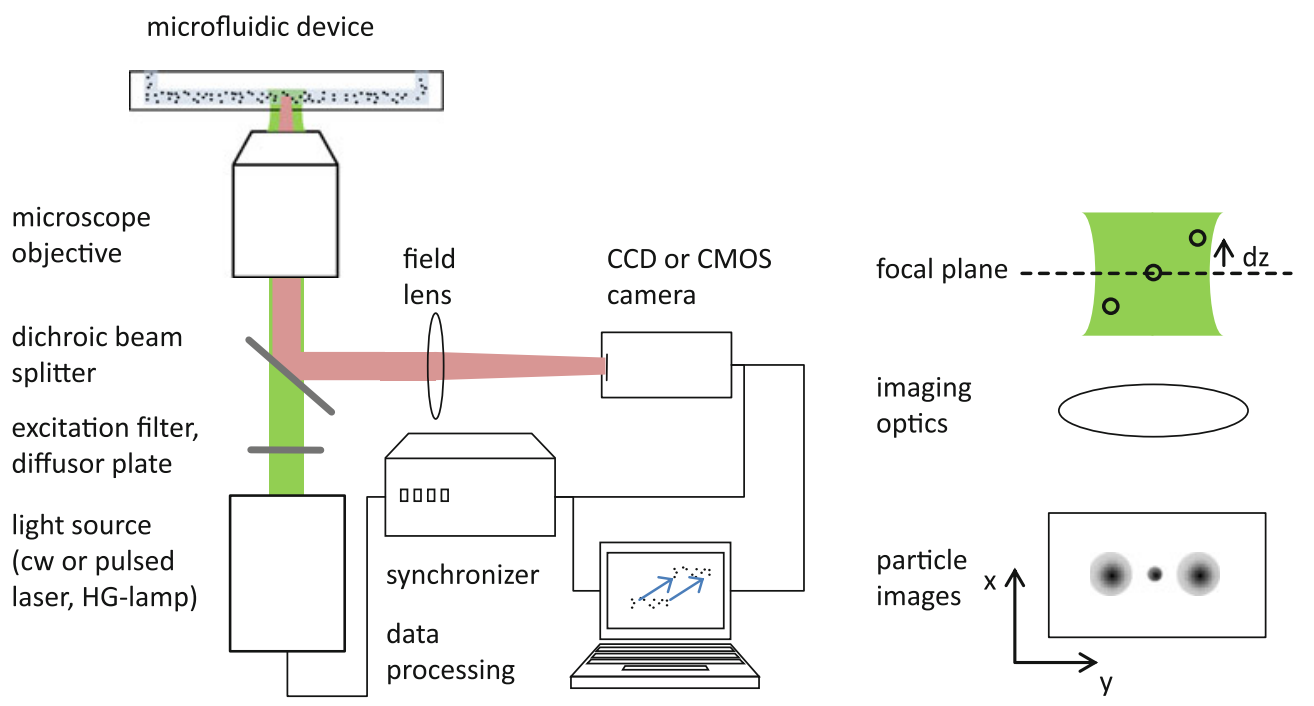

Fig. 1 Schematic of a typical $\mu \mathrm{PIV}$ system (left) and principle schematic of the imaging with reversed colors (right) 
assuming that out-of-focus particles with an intensity of $1 \%$ of the in-focus particle's intensity still cause a significant bias in the velocity estimate. Equation 1 can be rewritten (Rossi et al. 2011) as:

$$
\mathrm{DOC}=\frac{n_{w}}{n_{0}}\left\{\frac{(1-\sqrt{\varepsilon})}{\sqrt{\varepsilon}}\left(\frac{n_{0}^{2}}{\mathrm{NA}^{2}}-1\right)\left[d_{\mathrm{p}}^{2}+1.49 \lambda^{2}\left(\frac{n_{0}^{2}}{\mathrm{NA}^{2}}-1\right)\right]\right\}^{1 / 2}
$$

using the numerical aperture NA instead of the f-number to characterize the microscope objective and the factor $n_{w} / n_{0}$ to account for refraction through different media. As can be seen from Eq. 2, increasing the numerical aperture decreases the DOC. A high numerical aperture lens also resolves more details of the particle image. However, with increasing numerical aperture, the magnification usually increases as well, which is not always beneficial for the resolution in PIV (Kähler and Scharnowski 2011). In addition the field of view will be further limited and thus the dynamic spatial range decreases (Adrian 1997). On the other hand, smaller particles can decrease the DOC. Unfortunately, smaller particles will result in lower signal-tonoise ratios (SNR) thus reducing the accuracy of the method. Therefore, in standard $\mu$ PIV, the velocity estimate will always be biased by the DOC and the experimenter should know the DOC to determine the spatial resolution in depth direction and the introduced error due to spatial averaging.

Several attempts have been recently undertaken to use the information of defocused particles in the correlation plane. Erkan et al. (2008) used the temporal variation of the correlation peak height, which is related to a movement of the particles in the viewing direction, to measure the third velocity component. A decomposition of the distribution of the correlation coefficient is used by Fouras et al. (2009) to measure the $3 \mathrm{D} 2 \mathrm{C}$ velocity field. However, the method is very sensitive to noise due to its many assumptions (particle size, laser light intensity, imaging aberrations) and is only applicable for a large number of images, thus providing averaged velocity fields.

The improvement and adaptation of the $\mu \mathrm{PIV}$ technique to different pharmaceutical, biological or chemical applications is still difficult and an ongoing process. A report about different particle imaging techniques for microfluidic systems was already published in 2003 (Devasenathipathy et al. 2003) with the focus on image preprocessing and how to handle effects arising from the volume illumination. A more general overview of visualization techniques for micron scale flows is presented by Sinton (2004). Reviews of the state of the art of $\mu \mathrm{PIV}$ and its relevant applications were published by Lindken et al. (2009) and Wereley and Meinhart (2010). Guidelines for a proper experimental design in particular are given by Lindken et al. (2009) and Raffel et al (2007). However, the inherent limitations of standard $\mu$ PIV, using just one camera are

- The technique relies on volume illumination; thus the minimal thickness of the measurement plane is determined by the imaging optics' depth of focus.

- Since the whole volume is illuminated, out-of-focus particles will contribute to the cross correlation (depth of correlation) and, hence, introduce a bias in the measurements according to Eq. 2 (Olsen and Adrian 2000).

- The method is capable of measuring $2 \mathrm{C} 2 \mathrm{D}$ velocity fields only.

To overcome these limitations and to extend the velocity reconstruction to the third component, several methods have been proposed. Recent reviews about advanced methods, including some 3D techniques and proper experimental design can be found in Lee and Kim (2009) and Williams et al. (2010). However, the main focus of the paper at hand is to put in a common frame modern imaging methods that can be used for a later 3D reconstruction of the velocity field and compare their benefits and drawbacks. Therefore, scanning $\mu \mathrm{PIV}$ will be discussed in the following section. In paragraph 2 multi-camera approaches, i.e., stereoscopic and tomographic $\mu$ PIV will be shortly covered. Since single-camera techniques are often favorable due to the optical access limitation, as well as their robustness and reliable calibration, they will cover the main body of the paper. In this respect, confocal scanning microscopy (Sect. 3.1) is discussed in greater detail, followed by defocused particle imaging without aperture (Sect. 3.2), with a three-pinhole aperture (Sect. 3.3) and astigmatic imaging (Sect. 3.4). Taking also phase information into account, microscopic holography is presented in Sect. 3.5. Once the positions of the particles are known at two or more time instances, a variety of different tracking (PTV) and correlation-based (PIV) algorithms are suited for further processing. PTV has some advantages for the investigations of microfluidic devices. Therefore, a detailed comparative analysis of tracking and correlation-based approaches, including ensemble single pixel correlation, is presented in Sect. 4. The main findings of this study are then summarized in Sect. 5.

High-quality microscopy is crucial in biology and life sciences, and thus some methods for particle imaging were directly adopted from these disciplines to microfluidic engineering. Others were developed in 
parallel. However, we tried to find the original publications and recent studies in both fields, since both communities could get interesting ideas and valuable solutions to similar problems from each other.

\subsection{Epi-fluorescence scanning microscopy}

As discussed above, the standard $\mu \mathrm{PIV}$ system is able to measure the $2 \mathrm{D} 2 \mathrm{C}$ velocity field in a microfluidic device. It is possible to obtain the 3D3C information for steady and periodic flows, at least in an averaged sense, from a representation of $2 \mathrm{C} 2 \mathrm{D}$ slices.

Applying the incompressible continuity equation with the non-slip boundary conditions at the wall $(u, v, w=0)$ the third component can be calculated by integrating $\partial u_{i} / \partial x_{i}=0$ (Brücker 1995, 1997). Due to the volume illumination used for $\mu \mathrm{PIV}$, particles with large out-of-plane motion will remain in the images for successive illuminations. The resulting measured velocity vector will therefore be a projection of the true motion of the particles, hence decreasing the accuracy. The reconstruction of the 3D3C velocity field by scanning measurements in microflows was applied by several authors. Angele et al. (2006) used a rotating disk with glass plates of different thickness to quickly vary the optical path, and thus the depthwise position of the focal plane. They were able to reach a scanning speed of $100 \mathrm{fps}$ in the vertical direction with their system. The dual-plane PIV concept is able to measure the third velocity component (Raffel et al. 1996). However, in combination with scanning $\mu$ PIV measurements, it was applied by Shinohara et al. (2005) using a piezo actuator to move the microscope objective lens while recording, thus allowing for the reconstruction of the velocity in the whole volume. Unfortunately, the flow under investigation was unidirectional and the system was not tested in real 3D flow. Bown et al. (2005) used scanning in the depth direction to evaluate the flow in a ribbed channel geometry. The authors used the velocity data to later fit parabolic profiles and thus determine the position of the channel wall with an accuracy of $0.2 \mu \mathrm{m}$ in the depth direction. Rossi et al. (2009) used eight planes, each $2 \mu \mathrm{m}$ apart, for measurements in a microchannel with living cells on the wall. By the use of the velocity profiles in the direction of observation, the authors were able to extract the mean shear stress and the average surface topology of cells exposed to different shear strengths. In a later study (Rossi et al. 2010), a method was proposed to optimize the experimental parameters in order to decrease the error on the wall shear stress and topography measurement.

\section{Multi-camera recording approaches}

\section{1 (Scanning) Stereoscopic imaging}

Another way to obtain out-of-plane velocity is the observation from two different perspectives. Like for our own human 3D impression of the surroundings, at least two different views under an angle $\alpha$ are necessary. A review on stereoscopic imaging for PIV applications in macroscopic flows can be found in Prasad (2000). For microscopic applications, a recent description of the optical setups used can be found in Lee and Kim (2009a). Two different types of stereoscopic microscopes exist and are schematically shown in Fig. 2.

The Greenough type features two separated optics for each viewing direction and thus has a high numerical aperture and high optical resolution. However, large perspective distortions are the drawback. The more widely used systems for microfluidic investigations apply standard stereo microscopes and are of the common main objective (CMO) design. The benefit of these systems compared with the use of two different optics for each camera is the large overlapping in-focus region for the two views. However, since the light beams are not passing through the center of the large objective lens, they are asymmetrically distorted. Nevertheless, a recent comparison of both systems for velocity field measurements showed lower uncertainties using a CMO system (Yu et al. 2009). The CMO design is especially adopted for human sight and thus uses a large-diameter objective lens, through which both the left and right channels view the object at small angles between both views. The optical axis of the objective is normal to the object plane; therefore there is no inherent tilt of the image at the camera sensor plane. Due to the large and costly objective lens, systems are often limited in magnification to around $20 \times$. The left and right images are viewed by separated CCD cameras. Both cameras record the illuminated particles and the in-plane velocity vectors can be estimated using, for instance, PIV or PTV algorithms. Since the angle of observation is known by a calibration procedure, the out-of-plane velocity can be calculated in the overlapping region of both views. However, in a real system, the calibration is a complex procedure and suffers from distortions and aberrations induced by imperfections of the lens or by refraction between different media (e.g., glass and water of the microchannel). Especially, the small angle between the different views, typically $5-7^{\circ}$, is far from 


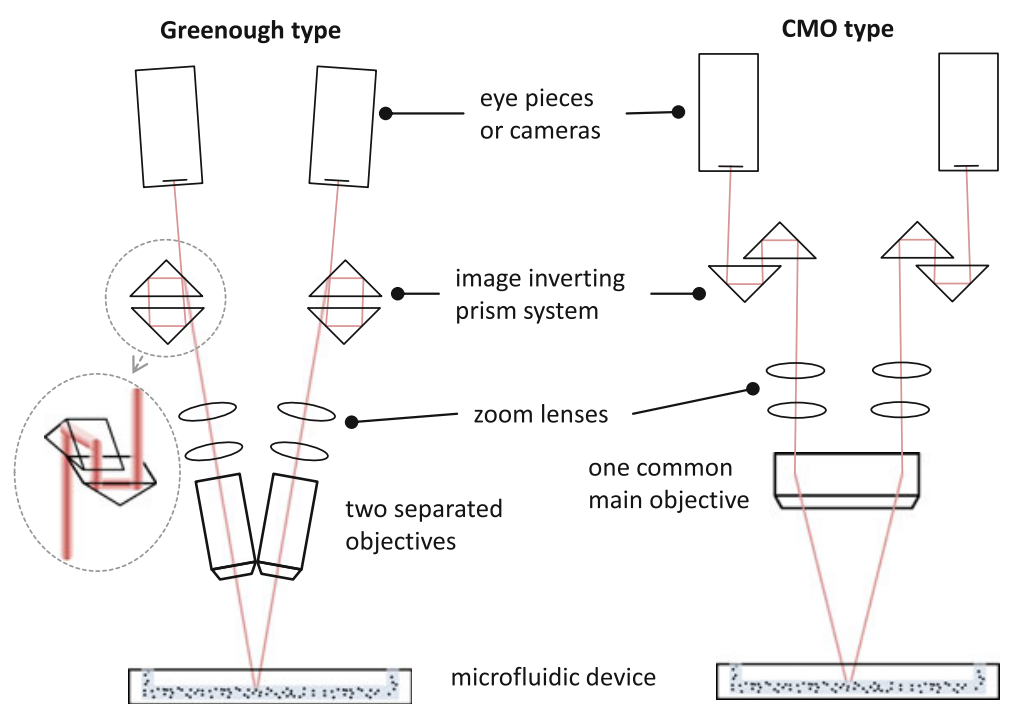

Fig. 2 Schematic of stereoscopic microscopes. Greenough type with two separated objectives (left) and the common main objective type (right), according to Lee and Kim (2009a)

being optimal ( $\geq 30^{\circ}$, Lawson and $\left.\mathrm{Wu}(1997)\right)$ and introduces errors in the estimation of the out-of-plane component. Giardino et al. (2008) made an uncertainty analysis for the determination of the displacement. They shifted microscope slides with particles stuck in all three directions and found an uncertainty of $\leq \pm 2 \mu \mathrm{m}$ for in-plane movements and $\leq \pm 5 \mu \mathrm{m}$ for the out-of-plane motion. These values are much smaller than the ones expected by the analysis of Lawson and Wu (1997) which match the experimental findings of many other authors that report a larger uncertainty.

To the authors' knowledge, the first application of the stereoscopic principle to measure the 3C2D velocity field in a microfluidic device was reported by Klank et al. (2002). Assuming a stationary flow, they used a single camera and obtained both perspectives one after the other. They scanned five planes with a distance of $10 \mu \mathrm{m}$. The later reconstruction of the velocity field in the volume and its comparison with numerical simulations showed reasonably good overall agreement, although no real calibration was performed. Klasen et al. (2005) and Lindken et al. (2006b) applied the technique to microfluidics using a stereo microscope and two cameras to record both images simultaneously, thus not requiring stationary flow. The big advantage of this procedure is the fact that the so-called self-calibration can be used to minimize the errors due to a misalignment of the system and calibration errors. Lindken et al (2006b) used a self-calibration procedure (Kähler et al. 1998; Kähler 2004; Wieneke 2005) to measure the three-dimensional flow in a T-mixer with a magnification of $23 \times$. In earlier measurements they reported an out-of-plane error nine times the in-plane error at an angle of $12.8^{\circ}$. Using an angle of $27.5^{\circ}$ the out-of-plane error was only four times larger than the in-plane error. A very interesting concept of increasing the angle between the two different camera views was recently presented by Hagsäter et al. (2008) and is schematically shown in Fig. 3. They applied a principle similar to Racca and Dewey (1988) and Arroyo and Greated (1991) who used an arrangement of mirrors to record both views with a single camera. For the microscopic version a mirror system consisting of a prism and two mirrors was designed, enabling an angle of $\sim 34^{\circ}$ for measurements in air and $\sim 26^{\circ}$ for measurements in a water-filled micro channel. The authors tested their system on a backward facing step. No sophisticated calibration procedure was used so far and the system was constructed for a magnification of $5 \times$, although it should also work for higher magnifications. However, total reflexion and the Fresnel effect will limit the maximum angle. Two mirrors under an angle of $90^{\circ}$ were used by Gazzola et al. (2009) to track single particles in an open capillary. However, due to image aberrations and the lack of a sophisticated calibration they were just able to visualize the evolving vortex flow.

Bown et al. (2006) presented a way to overcome the inherent problem of the limited depth resolution due to the depth of field. They used a super-resolution algorithm. The flow over a backward facing step was investigated using a stereo microscope with a magnification of $10 \times$. A prediction for the velocity is obtained via cross correlation. Later, a PTV algorithm was used to determine the velocity of individual tracer particles withour errors due to DOC. Since the position of the particles in the volume can be estimated quite well after a proper calibration, neither in-plane spatial averaging nor the projection of the velocity vector to 


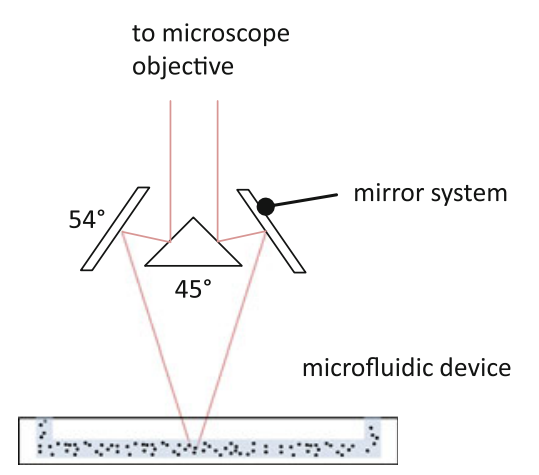

Fig. 3 Schematic of a mirror system to increase the angle between both views for stereoscopic particle imaging, according to Hagsäter et al. (2008)

the in-focus plane caused a bias the measurements. The data were later averaged on a grid of $10 \times 10 \times 10 \mu^{3}$ with significantly improved out-of-plane resolution and accuracy, especially in regions of strong gradients. However, although the accuracy of the determination of the third velocity component was greatly increased, a comparison of the continuity-based estimation of the out-of-plane component with stereoscopic imaging showed lower uncertainty for the continuity-based approach (Bown et al. 2007). Since multi-camera techniques suffer from calibration errors, scanning 2D measurements are a good alternative, although the problem of the DOC cannot be solved with epi-fluorescent 2D imaging.

The measurement of the out-of-plane velocity always suffers from the small viewing angle in CMO stereoscopic setups. Another main problem for stereoscopic $\mu \mathrm{PIV}$ arises from the complex calibration procedure required. Imperfections in the lens system and slight misalignment result in the misalignments of the focal planes inside the measurement volume (Cierpka et al. 2011b). Since the third component is reconstructed using the in-plane results with the assumption of the coinciding focal plane, the errors for the out-of-plane increase strongly, especially in regions of strong gradients. An additional inherent problem is the large depth of field for the CMO lenses and thus the large DOC. It is therefore nearly impossible to get unbiased data with a stereoscopic CMO setup.

\subsection{Tomographic imaging}

A real volumetric method is the tomographic PIV or PTV. For the velocity estimation, the volume distribution of the particles is reconstructed from the images, recorded from different viewing directions. The velocity field is then calculated by either a volumetric cross-correlation or a volumetric tracking algorithm as opposed to stereoscopic approaches, where the third component is reconstructed by the in-plane velocity. At least four different views are required for a reconstruction with sufficient quality for most applications (Elsinga et al. 2006). The reconstruction of the volumetric intensity field is a mathematically ill-posed problem and iterative algorithms have to be used. Multiplicative algebraic reconstruction techniques (MART), starting from a uniform nonzero intensity in the whole volume are simple methods frequently used for the reconstruction. The volume is separated in cuboid volume elements, the so-called voxels, and the intensity for each voxel is iteratively updated according to the boundary conditions. Usually, five or more iterations are necessary to reach a reasonable accuracy in the reconstruction. However, the reconstruction is highly costly in terms of computational time and the quality strongly depends on the calibration error. The error should be lower than 0.2 pixels in the whole volume for a good quality reconstruction. In order to decrease computational time, different methods to get an initial solution or speed up the calculation were proposed. The main benefit of this method is that not all voxels have to be updated from the beginning. Worth and Nickels (2008) were able to reduce the computational time by a factor of 2.5 using a multiplicative first guess. Atkinson and Soria (2009) used a simultaneous MART with a multiplicative line-ofsight estimation of possible particle location and achieved a factor of up to 77 increase in speed with the same accuracy. A very interesting finding of the authors is that for practical applications the accuracy is limited to 0.22 pixels for in-plane components and 0.3 pixels for the out-of-plane component. By assuming an particle image displacement of 10 pixel this leads to an dynamic velocity range of DVR $=10 / 0.22 \approx 45$. Although this value is relatively low compared with other measurement techniques, it is often sufficiant to visualize the large-scale features of the flow. Maas et al. (2009) proposed a projective transformation-based approach to reconstruct the particle positions in the volume and later to use a 3D least squares tracking 

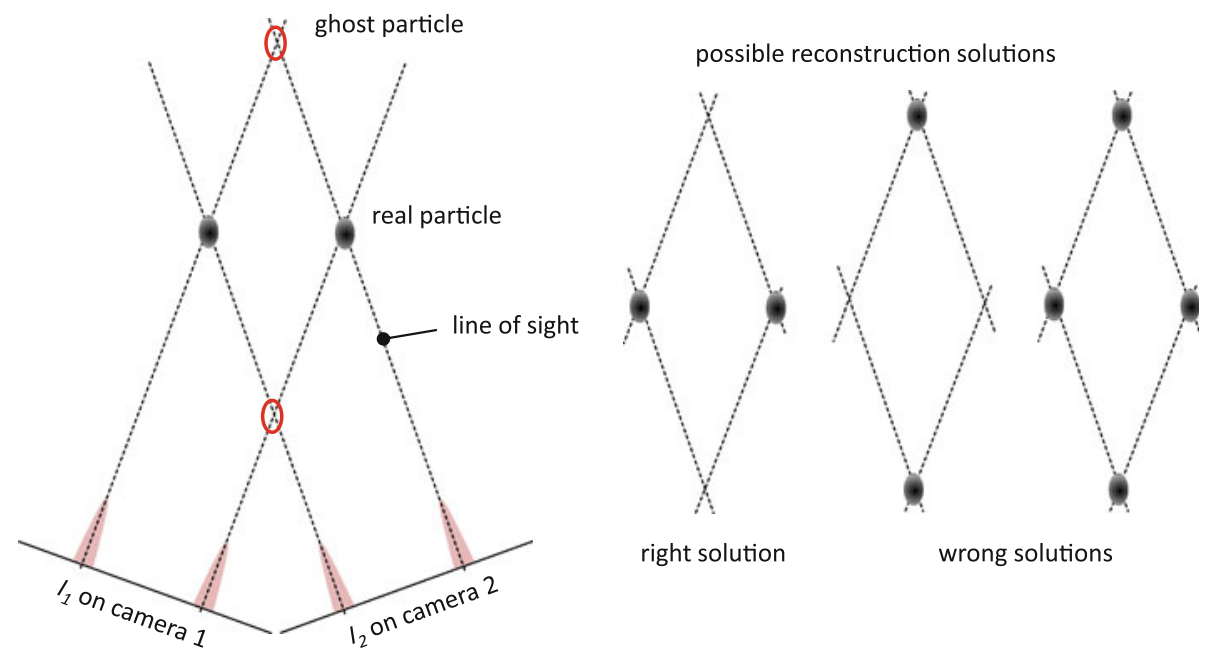

Fig. 4 Schematic of the tomographic imaging and the appearance of ghost particles (left) and possible solutions of the reconstruction out of the two camera images (right), according to Elsinga et al. (2011)

instead of cross correlation to estimate the velocity and rotation as well as shear information. However, a major difficulty for the tomographic reconstruction is the appearance of the so-called ghost particles arising from the mathematically ill-posed problem as illustrated in Fig. 4. The number of ghost particles increases with the number of real particles and thus limits the seeding concentration to 0.05 particles per pixel, which is one order of magnitude lower than for 2D PIV. Since a certain amount of particles is required in an interrogation volume, also the dynamic spatial range of scale, defined as mean value of the ratio between the length of the whole volume $L_{x, y, z}$ and the corresponding length of an interrogation volume $L i_{x, y, z}=\mathrm{DSR} \approx$ 12 (Elsinga et al. 2006) is often low. The ghost particles are usually of lower intensity but will contribute to the velocity estimate. If ghost particles occur from the same set of particles in both frames, their displacement corresponds to the averaged displacement of the actual particles causing them (Elsinga et al. 2011). Therefore, ghost particles will always bias the velocity estimate and great care has to be taken to achieve the proper seeding concentration in tomographic PIV experiments.

Using a stereo microscope, two different views are available. To the authors' knowledge the first attempt to reconstruct the particle distribution in a micro volume was presented by Lindken et al (2006a). The velocity estimation suffered from the elongated reconstruction of the particles due to the small viewing angles of the stereoscopic setup as schematically shown in Fig. 4. Viewing angles of $20^{\circ}$ were reported by Kim et al. (2011), who used a self-made microscope and applied four cameras to investigate the flow inside a droplet on a moving surface tomographically. The measurement volume was $2.5 \times 2.1 \times 0.4 \mathrm{~mm}^{3}$. To decrease the amount of ghost particles, sparse seeding was used and 25 successive reconstructions of the $3 \mathrm{D}$ distribution of tracer particles were added to synthetically increase the seeding concentration and to allow for volumetric cross correlation. Currently this limits the technique in microfluidics to stationary flows. The authors used a continuity-based approach to estimate a global uncertainty for all directions, which was reported to be 0.2 pixel. This corresponds to $0.086 \mathrm{~m} / \mathrm{s}$, which is about $8.6 \%$ of the moving velocity of the surface.

In general, tomographic PIV might be used more frequently for certain scientific applications. However, despite the necessary availability of more than one optical access, the need for a precise calibration of the two or more cameras in the common field of view is the major drawback of multi-camera techniques. Methods to record the 3D particle distribution in a volume with a single camera are therefore more attractive in microfluidics such as the fast point-wise scanning of the volume as outlined next.

\section{Single-camera recording approaches}

\subsection{Confocal scanning microscopy}

In comparison to epi-fluorescent imaging, confocal scanning microscopy is an approach to dramatically increase the out-of-plane, as well as the in-plane resolution. This is achieved using point-wise illumination. Point-wise illumination was generated by using a lamp and an aperture at the time it was invented (Minsky 
1961). Nowadays, focused laser beams are most often used for the illumination and the technique is often referred to as confocal laser scanning microscopy (CLSM). In Fig. 5, the principle schematic of a confocal laser scanning microscope is drawn. The laser light is focused by a micro lens onto the sample. This microlenses are often arranged on a rotating disk to increase scanning speed. However, focusing at a certain time instant result in just a small part of the sample being illuminated. Another pinhole aperture (typically several microns) is used to filter light that is coming from outside the focal point. These pinholes are also equally spaced and arranged in a spiral on a spinning disk, which is called Nipkow disk after its inventor. The mechanical connection of both disks allows for an easy and precise synchronization. A photodetector or a camera can then be used to record the fluorescence intensity at the focused point. To record a whole 2D representation of the sample, data points in the $x y$-direction have to be scanned. The technique is widely used in biology, where the contrast of the images and the increased resolution was necessary to observe certain micro organisms. The calibration of the system can be performed in the same way as for epifluorescence imaging with a grid or other well-suited object.

The technique was first used in micro fluid mechanics to investigate the mixing process in micro mixers (Ismagilov et al. 2000; Gösch et al. 2000). Numerical results for the cross-sectional interfaces between two fluids from different inlets in a curved micro mixer were validated against experimental results obtained using fluorescein and confocal microscopy. In order to measure velocities the technique was first applied to $\mu$ PIV by Park et al. (2004). Conventional methods for scanning used galvanic or piezoelectric mirrors to scan the probe volume. However, the scanning speed was usually limited to several frames per second. The scanning time was decreased considerably by the Nipkow disk and a rotating disk with microlenses to focus the laser. Typical full-field scanning rates were $50 \mathrm{~Hz}$ (Lima et al. 2006), $120 \mathrm{~Hz}$ (Park et al. 2004), $200 \mathrm{~Hz}$ (Lima et al. 2007) up to $4,800 \mathrm{~Hz}$ (Kinoshita et al. 2007). Liebling et al. (2006) used a slit instead of pinholes to record a whole line of the sample at once. They achieved $120 \mathrm{fps}$ with their system, enabling them to measure the fast cardiac motions of a beating embryonic zebrafish heart.

The application of these techniques to velocity field measurements has the benefit that the images of particles viewed by confocal microscopy show a significant increase in signal-to-noise ratio. The thickness of an optical slice is in the order of $1 \ldots 2 \mu \mathrm{m}$ and light from out-of-focus particles does not decrease the image quality and thus no bias in the velocity measurement is introduced. Klein and Posner (2010) showed a large increase in vector yield using confocal scanning $\mu \mathrm{PIV}$ in comparison with wide field $\mu \mathrm{PIV}$. Confocal scanning microscopy shows better performance especially for larger volume fractions $(>0.45 \%)$ of particles in the fluid. Scanning confocal microscopy was successfully applied to microscopic flows of evaporating droplets (Kinoshita et al. 2007), biological cell flows (Lima et al. 2006, 2007), and capillary flows (Park et al. 2004). Ichiyanag et al. (2007) combined laser-induced fluorescence (LIF) for the measurement of the $\mathrm{pH}$-value with $2 \mathrm{D} \mu \mathrm{PIV}$. They used a rotating disk confocal microscope for increased depth resolution. The dye for the LIF measurements was fluorescein and showed an emission maximum at a wavelength of $\lambda=645 \mathrm{~nm}$. The tracers used for PIV were $1 \mu \mathrm{m}$ polystyrene particles, which had an emission maximum at

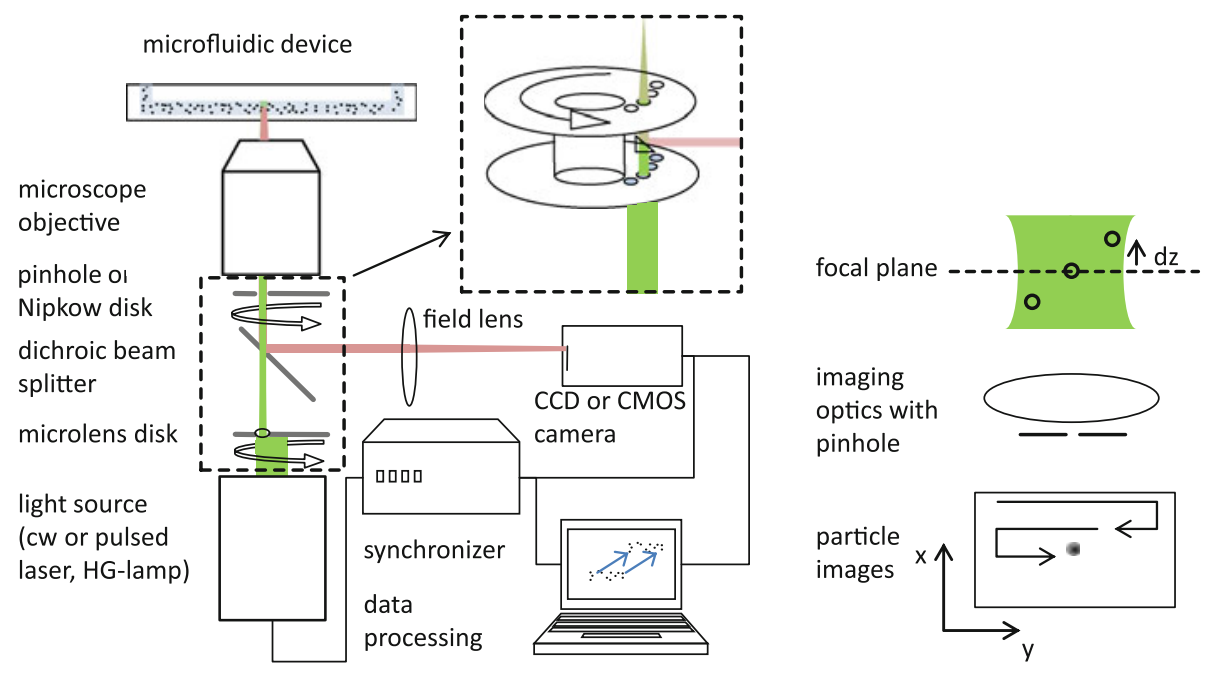

Fig. 5 Schematic of a confocal laser scanning $\mu$ PIV system with a spinning disk system after (Lima et al. 2006) (left) and principle schematic of the imaging with reversed colors (right) 
a wavelength of $\lambda=518 \mathrm{~nm}$. They were able to capture images for the evaluation of the $\mathrm{pH}$-value and the velocity separately, using a 3CCD color camera. Hoffmann et al. (2007) applied scanning standard epifluorescence microscopy to measure the 2D2C velocity field in a T-mixer and CLSM to measure concentration via LIF. The third velocity component was estimated using the continuity approach as outlined in Sect. 1.2.

The limitations of the confocal microscopy are the expensive lab equipment and further more the limited temporal resolution. The minimal time distance between images is restricted by the scanning speed. In Fig. 6, the necessary time resolution $\Delta t$ is given versus the fluid velocity $u$ for different magnifications, similar to the analysis by Hain and Kähler (2007). The graph is based on the assumption of a 10-pixel particle image displacement on the image for simplicity. The Reynolds number given on the upper horizontal axis should provide a first estimate for the user and is based on a length scale of $100 \mu \mathrm{m}$ and the kinematic viscosity of water.

The region highlighted in light gray indicates the use of CCD cameras with a scanning rate lower than $10 \mathrm{~Hz}$ or a minimal time interval between two frames of $0.1 \mathrm{~s}$. It is evident from Fig. 6 that this kind of equipment is useful for low Reynolds number investigations $(R e=O(0.01 \ldots 0.1))$ and low magnifications. Using high-speed cameras and/or high-speed CLSM increases the range for measurements to $R e<5$. For higher Reynolds numbers, pulsed light sources and double-frame cameras have to be applied (white region).

However, with increasing scanning speed, confocal microscopy shows a great potential for further use in $\mu \mathrm{PIV} / \mathrm{PTV}$, since the problems due to low SNR and DOC can be avoided successfully.

\subsection{Techniques based on out-of-focus imaging without aperture}

On the right-hand side of Fig. 1 the images for particles beyond the focal plane are drawn. It is evident that the image diameter depends on the distance from the focal plane. A model to describe the defocused imaging of particles in microscopes was developed by Olsen and Adrian (2000). They assumed a Gaussian

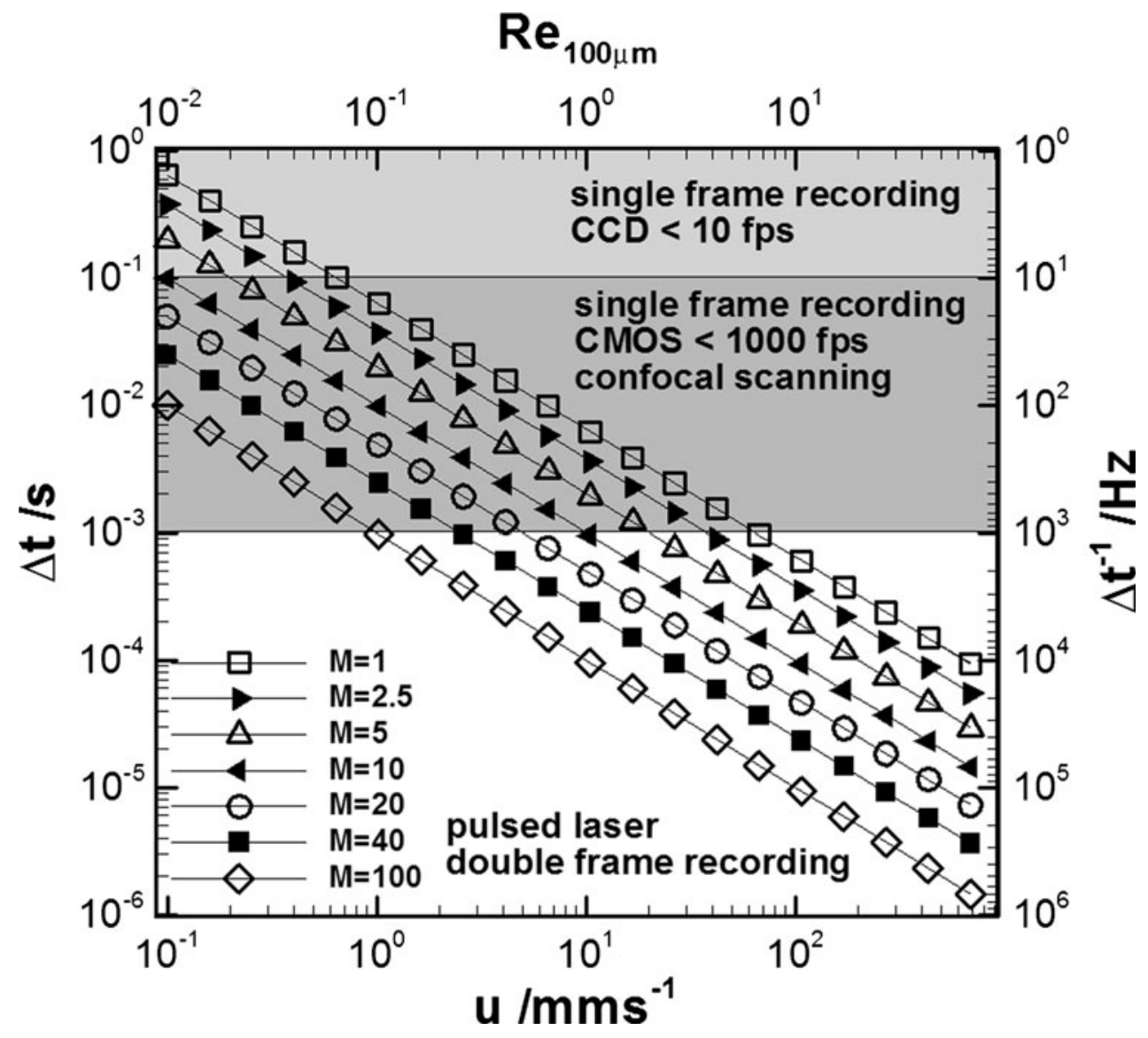

Fig. $6 \Delta t$ vs. $u$ for different magnifications 
intensity distribution for the particle images. Contributions are due to the diameter of the particle itself (geometric part), diffraction and defocusing. The diameter $D_{\mathrm{I}}(z)$ of a particle image can now be described as a function of the distance to the focal plane $z$, with the assumption that the working distance of the lens is significantly larger than $z$. This surely holds for most microscopes where the working distance of the lens is typically at least one order of magnitude larger than the channel width. The diameter is given by the following equation (Meinhart and Wereley 2003; Rossi et al. 2011b):

$$
D_{\mathrm{I}}(z)=M \sqrt{d_{\mathrm{p}}^{2}+1.49 \lambda^{2}\left(\frac{n_{0}^{2}}{\mathrm{NA}^{2}}-1\right)+4 z^{2}\left(\frac{n_{0}^{2}}{\mathrm{NA}^{2}}-1\right)^{-1}}
$$

where $d_{\mathrm{p}}$ denotes the particle diameter, $\lambda$ is the wavelength of the emitted light, $n_{0}$ the refractive index of the immersion medium of the lens, and $M$ and NA the magnification and numerical aperture of the lens, respectively. However, recent investigations showed differences in experimental data, especially for high NA objective lenses (Rossi et al. 2011b; Kloosterman et al. 2010). Nevertheless, the diameter of the particle image can be correlated with its depth by a proper calibration.

One of the first applications of defocused images to determine the out-of-plane velocity was realized by Stolz and Köhler (1994). They used the size of the defocused particle images to estimate the third velocity component by PTV in a $1.5 \mathrm{~mm}$ thick light sheet. The mean value of the velocity in the axial direction, in a laminar channel flow, was just 5\% higher than the reference measurement by laser Doppler anemometry, although the standard deviation was $10 \%$. The same principle was also applied for macroscopic flows by Murata and Kawamura (1999), who reported an rms error for the out-of-plane position of the particles' position below $5 \%$ of the measurement depth.

Hiraoka et al. (1990) analyzed the three-dimensional optical transfer function of particles to determine the three-dimensional structure of biological specimens with an epi-fluorescence microscope. For microscopic flows, a method taking advantage of the size of diffraction rings using forward scattering of light was developed by Ovryn and Hovenac (1993) and applied to a microchannel of $315 \mu \mathrm{m}$ in depth to measure the velocity profile in the depth direction. However, for a good SNR, the particles had a diameter of $7 \mu \mathrm{m}$ and showed a tendency to sink in the fluid. Only the lower part of the profile could be measured. Guerrero et al. (2000) extended the method to backward and side scatter configurations and compared experimental results with Lorentz-Mie theory. In a later study (Guerrero-Viramontes et al. 2006) the method was extended, also including Huygens-Fresnel light propagation. However, the measurement errors for the position are relatively large (up to 30\% of the volume). Recently, Moreno-Hernandez et al. (2011) used wavelet-based noise reduction and the central spot size of the interference pattern. The reported errors are still very high, especially for larger particles $\left(25 \%\right.$ with $\left.d_{\mathrm{P}}=15 \mu \mathrm{m}\right)$. The measurement technique, in general, relies on similar principles as in-line holography. A theoretical assessment of the method's uncertainty for 3D PTV regarding particle and pixel size, magnification and resolution was done by Padilla Sosa et al. (2002). Speidel et al. (2000) applied the method for the reconstruction of $216 \mathrm{~nm}$ beads in an agarose gel. Using an oil immersion lens with a magnification of 100 , they were able to measure the bead position with subnanometer precision within a volume of $3 \mu \mathrm{m}$ in depth. The threedimensional movement of coli bacteria in a volume of $481 \times 335 \times 65 \mu \mathrm{m}^{3}$ was measured by Wu et al. (2005) using the defocused bacteria images with a conventional microscope and illumination by a mercury lamp. For calibration, $1 \mu \mathrm{m}$ particles were used. Surprisingly, it was observed that the appearance of the diffraction rings was not symmetric around the focal plane, even though it should from theoretical predictions. They concluded that spherical aberrations of the lens system were the reason for this. Measuring the size of the outer diffraction ring and comparing it to a theoretical model, Luo et al. (2006) and Luo and Sun (2011) measured the Brownian motion of particles and Park and Khim (2006) were able to measure the 3D3C flow around a sphere $(95 \mu \mathrm{m}$ in diameter) in a microchannel of the same height. The particles used had a diameter of $500 \mathrm{~nm}$. The uncertainty reported for a single-point measurement was $7.6 \%$ of the mean velocity and decreased for ensemble averaged data to $5.6 \%$. Since the apperance of the diffraction rings is largely dependent on the refractive index of the medium, Park et al. (2010) used the diameter of the outmost diffraction ring of particles, stuck outside of a chanel to determine the refractive index of the medium in the channel and thus the concentration of the fluid.

Peterson et al. (2008) measured the velocity profile in a $50 \mu \mathrm{m}$ deep channel by manually identifying particles and determining the size of their outer diffraction ring. They found particles with a diameter of $3 \mu \mathrm{m}$ sufficient for the later data processing. A large uncertainty arises for the technique due to the short illumination time of the small particles $(100 \mathrm{~ms})$ and the low SNR consequently. The determination of the 
diffraction rings' sizes is thus very complicated. Despite the need of a good knowledge in image processing, the low particle image density $(\approx 2.25$ per frame) is the major drawback of the technique. Another problem of the technique is its symmetry to the focal plane. If the focal plane is within the sample, the distance from the focal plane can be measured, but not if the particle is located above or below the focal plane. The unambiguity in the depth coding can be achieved by breaking the axis symmetry of the imaging optics. Toprak et al (2007) applied a method they called bi-focal imaging. Using a beam splitter, the fluorescence signal was split onto two optical paths. $30 \%$ where imaged with an additional lens, resulting in a focal plane $1 \mu \mathrm{m}$ from the original focal plane of the microscope. Evaluating both the defocused and focused images, they were able to follow $200 \mathrm{~nm}$ beads with a standard deviation of $\sigma<4 \mathrm{~nm}$ for a $z$-range of $600 \mathrm{~nm}$.

Another method to detect particle depth positions is the use of diffraction gratings (Towers et al. 2006; Dalgarno et al. 2010). Evaluating the defocused particle images for different orders of diffraction, it is possible to determine the depth position. Angarita-Jaimes et al. (2006) reported an uncertainty of $8 \mu \mathrm{m}$ over a depth range of $0.2 \mathrm{~mm}$. The disadvantage of this technique is that three particle images are produced, thus decreasing the effective sensor size.

\subsection{Defocused imaging with aperture (three-pinhole technique)}

The depth coding via a three-pinhole aperture is a method that applies defocused particle imaging with an optical spatial filter. This method was proposed by Willert and Gharib (1992) and applied for macroscopic two phase flow by Pereira et al. (2000) and Pereira and Gharib (2002). A theoretical examination of the underlying optics for macroscopic applications was presented by Kajitani and Dabiri (2005, 2008). Later the particle characterization was improved by a new imaging volume definition (Grothe and Dabiri 2008).

Yoon and Kim (2006) successfully adopted the technique to microfluidics. The principle schematic is given in Fig. 7. In contrast to the original $\mu$ PIV setup, shown in Fig. 1, an aperture mask, featuring three pinholes is introduced directly after the objective lens. Since the aperture mask would block a large amount of the laser light, usually the illumination is shifted to shine onto the sample from another axis, thus requiring transparent samples. On the right side of the same figure, the imaging of particles in the volume is schematically shown. For a complete treatment of the geometric optics using a microscope, the interested reader is referred to Yoon and Kim (2006). Recently, the calibration procedure was extended to interfaces with different index of refraction (Yoon et al. 2011). Interestingly, also the inverse principle, measuring the refractive index from the triplet of a bright spot, integrated on a microchannel, was successfully applied (Yoon and Yang 2010). In general, an out-of-focus particle is imaged as a triplet depending on its position in the volume. In the focal plane, a single image will be detected on the sensor. Depending on weather a particle is in front or behind the focal plane, the arrangement of the triplet is mirrored, allowing for an unambiguous determination of the particle's position. For the out-of-focus particles, the distance between the edges of the imaged triplet is directly related to the distance from the focal plane. Image preprocessing must now be applied to detect corresponding spots, forming a triplet. A circumcircle (diameter $d_{i}$ in Fig. 7) can be fitted through the single spots. Its center is related to the particles' position in the $x y$-plane. The
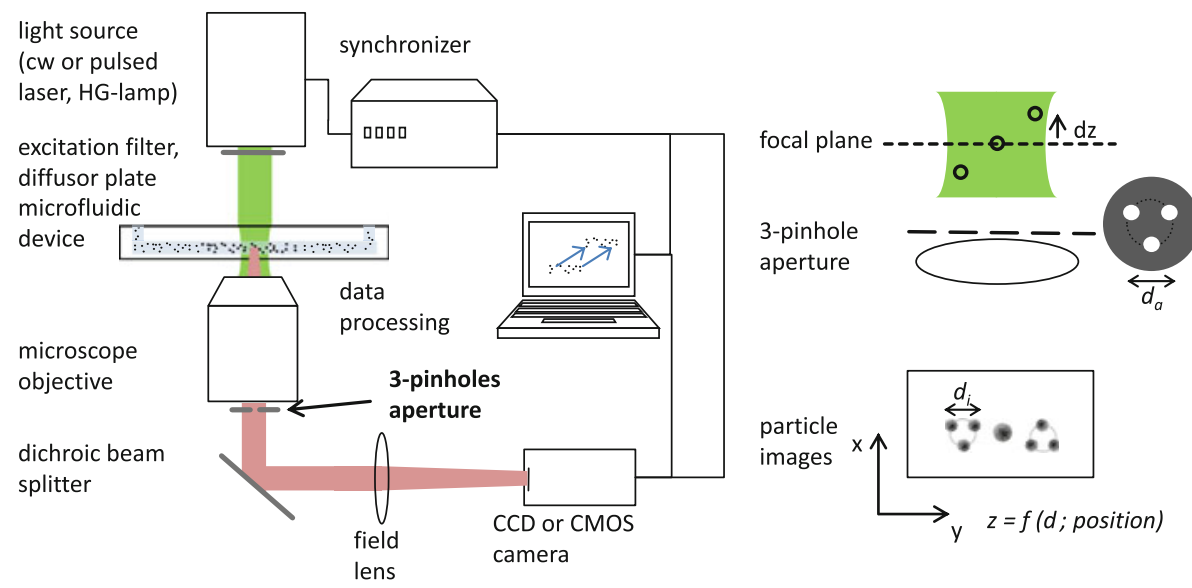

Fig. 7 Schematic of the three-pinhole defocusing technique (left) and principle schematic of the imaging with reversed color (right) 

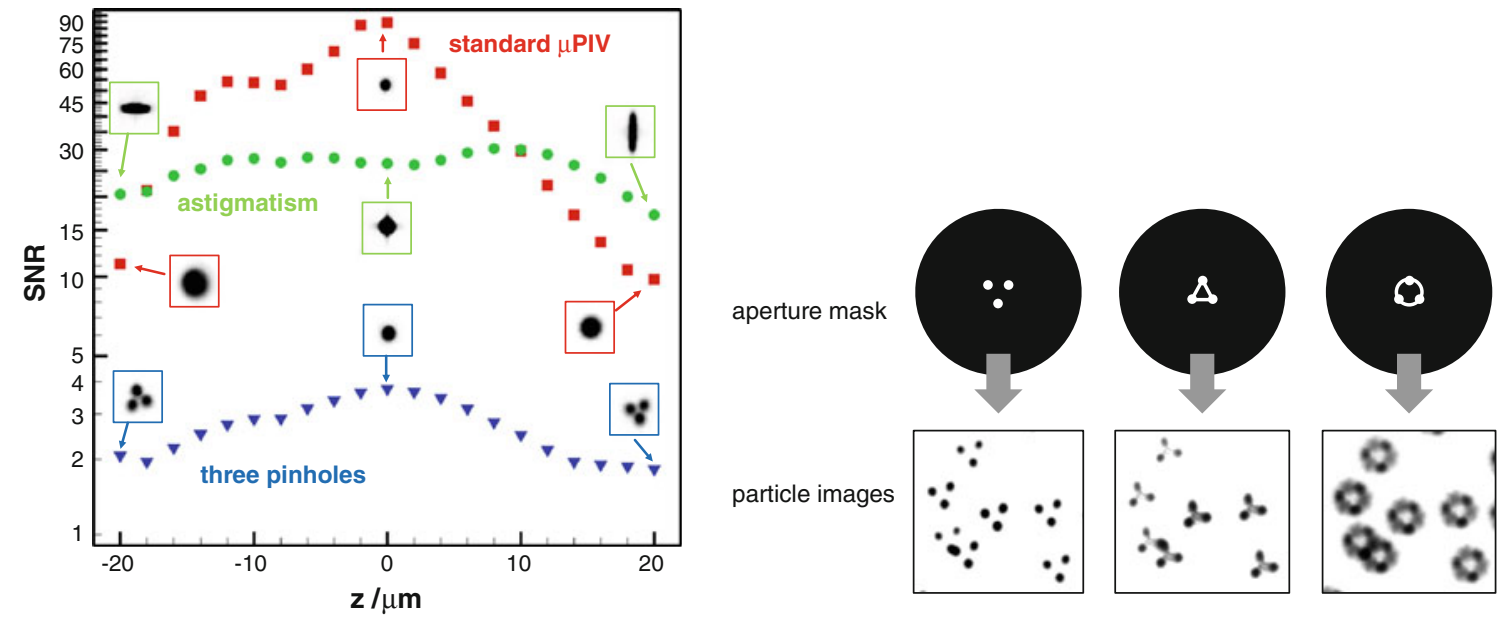

Fig. 8 Comparison of the signal-to-noise ratio (SNR) for standard imaging, the three-pinhole method and astigmatic imaging (left). Particle images for different apertures masks (right)

diameter $d_{i}$ of a triplet can be calibrated to the actual z-position of the particle and increases linearly with distance from the focal plane for microscopic imaging. Changing the circumcircle for the aperture mask $d_{a}$ will also change the slope of $\mathrm{d} d_{i} / \mathrm{d} z$. The lager the diameter at the aperture $d_{a}$, the larger the slope $\mathrm{d} d_{i} / \mathrm{d} z$. Thus, the measurement depth and the sensitivity and resolution of the system can easily be adopted to different requirements. Since no additional elements, such as lenses or diffraction gratings are introduced, the benefit of high-quality microscope objectives with low image aberrations is preserved. Therefore, the calibration for the $z$-direction holds for the entire sensor $\left(\mathrm{d} d d_{i} / \mathrm{d} z=\right.$ const.). For astigmatic imaging, in comparison, a correction due to lens aberrations has to be applied. Another advantage of such a system is that the error of the particle image detection algorithm is spread over three images.

The low seeding rates and the low light intensity due to the small pinholes are the major limitations of this technique. The SNR, in this respect the maximum intensity divided by the background noise is shown in Fig. 8 for standard imaging, the 3-pinhole method and astigmatic imaging. The pinholes had a diameter of $1.5 \mathrm{~mm}$, the diameter of the circumcircle was $4 \mathrm{~mm}$. The magnification was $20 \times$. These were the same conditions used by Yoon and Kim (2006). A glass plate with $5 \mu \mathrm{m}$ fluorescent particles from Microparticles $\mathrm{GmbH}$ was illuminated from the top by an optical fiber connected to a diode pumped cw-laser with an output power of $1 \mathrm{~W}$. To reach approximately the same measurement depth, a cylindrical lens with $f=150$ $\mathrm{mm}$ was used for the astigmatic imaging. The images were recorded with a Sensicam QE from PCO with an exposure of $10 \mathrm{~ms}$ to reach almost full-well capacity for in-focus particles with standard imaging. The SNR for standard imaging shows the highest values of SNR $\approx 90$ at the focal plane at $z=0 \mu \mathrm{m}$ and decreased to $\mathrm{SNR} \approx 10$ at a distance of $z= \pm 20 \mu \mathrm{m}$ from the focal plane. The astigmatic imaging is a technique that works without masking the optics and thus allows more light to reach the sensor. The principle will be explained in detail in the next section; however, the graph shows two peaks in the maximum intensity where the two distinct focal planes lie (Cierpka et al. 2011a). The SNR is much lower than for standard imaging and decreases from around SNR $=30$ to 15 for out-of-focus imaging. Using the 3-pinhole mask, a large portion of the light is filtered out and the SNR reaches a maximum of SNR $=4$ in the center and decreases to around SNR = 2 for out-of-focus regions. The results give an estimate for the image quality that can be expected for the different techniques. Applying astigmatic imaging the SNR decreases by a factor of 3 and using the pinhole aperture it decreases by a factor of more than 20 compared with standard imaging. Min and Kim (2011) recently used large particles $(5 \mu \mathrm{m})$ and an image intensifier to increase the image quality. They combined the defocused PTV method in the far field with total internal reflection microscopy close to the wall and reported a decreased uncertainty for the estimation of the wall gradients.

However, if the image quality is sufficiently high, it is often difficult to determine particle images that correspond to a triplet. For this reason masks with different patterns can be used to improve this procedure. On the right-hand side of Fig. 8 the particle images are shown for the standard mask, a mask where the pinholes are connected by lines and a mask with a circle connecting the pinholes. It is evident from the images that is is much easier for data processing to find corresponding triplets if they are somehow connected. All kinds of masks are possible. Additionally, image preprocessing correlation with a virtual 
mask (Nasarek 2010) might help to reliably detect triplets. Other possibilities include using a color camera and color filters in front of each pinhole in the aperture mask (Tien et al. 2008), or using an annular aperture (Lin et al. 2008), or the known active rotation of an off-axis aperture (Rohály et al. 2001).

To the authors' knowledge Yoon and Kim (2006) were the first using the technique to investigate the flow over a backward facing step in a volume of $768 \times 388 \times 50 \mu^{3}$. From geometric optics it is evident that the center of the circumcircle does not directly correspond to the particle position in the $x y$-plane. For the correction of the $x y$-position, a polynomial fit was used to map the final data. The calibration was done by moving a glass plate with particles in the axial, i.e., $z$-direction using a translation stage. In order to take into account the change of refractive index, the same glass plates and the same fluid was used. The authors used a high-speed camera at a recording rate of $1000 \mathrm{fps}$ to measure time-resolved particle trajectories, restricting the experiment to a Reynolds number based on the hydraulic diameter of $R e=0.025$ (cmp. Fig. 6). To overcome the problems due to low SNR, relatively large particles of $3 \mu \mathrm{m}$ were used.

To study the flow inside an evaporating water droplet, Pereira et al (2007) applied the technique to a volume of $400 \times 300 \times 150 \mu \mathrm{m}^{3}$. A high-speed CCD camera $(\sim 250 \mathrm{fps})$, using 16 separated areas on the chip was used. Thus a background image, taking into account the different gain for all 16 areas, had to be removed. An extensive error analysis is reported. Finally, taking all sources of errors into account (calibration stage resolution, processing algorithm, pixel size), the authors reported an error lower than $3 \%$ $(3.3 \mu \mathrm{m})$ for a depth range of $110 \mu \mathrm{m}$. The authors were able to succesfully measure the particle trajectories with flow velocities up to $1.5 \mathrm{~mm} / \mathrm{s}$ (cmp. Fig. 6). Lu et al. (2008) applied the method later for in-vivo measurements of the beating heart of an embryonic zebrafish. The authors used an aperture with $d_{a} \approx 4$ and a pinhole size of $2 \mathrm{~mm}$ to image $1 \mu \mathrm{m}$ fluorescent beads. For the recording an $\mathrm{Ar}^{+}$-laser was used in combination with a high-speed camera. They were able to reconstruct the movement of the ventricle by recording images of trapped particles at the wall during a full cardiac cycle. Nasarek (2010) measured the flow of a toroidal micro vortex in a channel with $50 \mu \mathrm{m}$ in depth. The aperture triplet had pinholes with a diameter of $1 \mathrm{~mm}$ and a circumcircle with a diameter $d_{a}=4 \mathrm{~mm}$. The particles used had a diameter of $1 \mu \mathrm{m}$ and were imaged with $20 \times$ magnification. The author mentioned that due to the long exposure time, trails at the particle images appeared which negatively affected the accuracy. Nevertheless, using advanced image preprocessing, the errors were reported to be $2 \mu \mathrm{m}$ for the in-plane position and $3.8 \mu \mathrm{m}$ for the out-of-plane position. Although the technique is widely used, it has the drawback that a big part of the fluorescence signal is blocked by the aperture lens. A technique that uses the whole incoming light is astigmatic imaging.

\subsection{Imaging based on aberrations (Astigmatism)}

Astigmatism or anamorphic imaging is a way to break the axis symmetry of an optical system thus allowing for the depth coding in 2D images. The word astigmatism is based on the Greek description for not $(a-)$ point-shaped (stigma). An optical system that features astigmatism has two focal lines instead of a focal point and the astigmatic image of a point source is thus an ellipse. The principle is schematically shown in

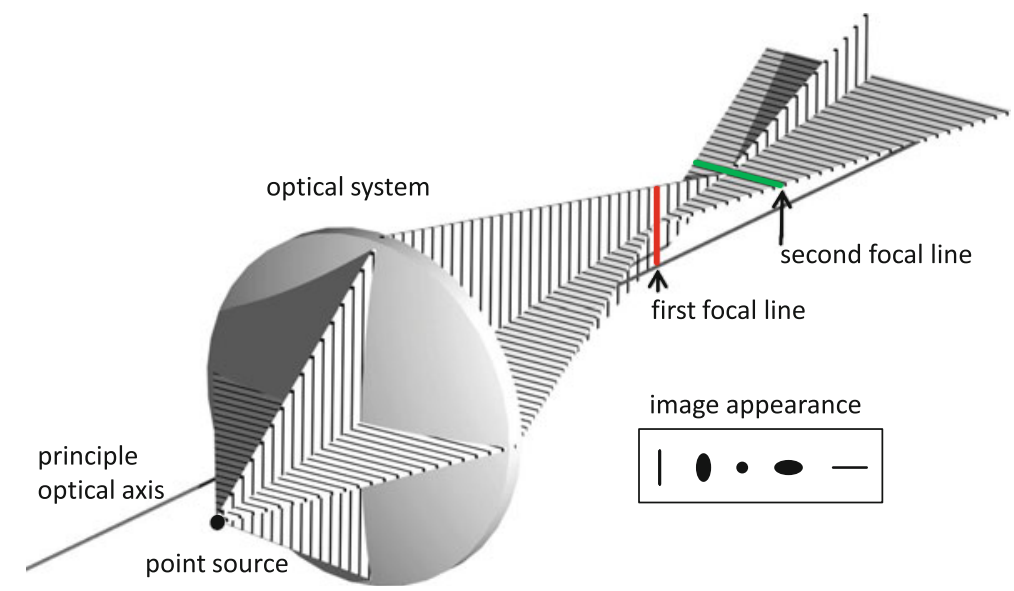

Fig. 9 Schematic of astigmatism caused by off-axis imaging and the appearance of the image at various distances between the two focal planes, according to Hain et al. (2009) 
Fig. 9. The off-axis imaging of a point source can already cause astigmatism. Due to the different refraction that takes place in the horizontal and the vertical directions of a lens looked upon at an angle two, focal lines perpendicular to each other are produced. The image of the point source is first a vertical line and changes gradually into a defocused circle and later to a horizontal line at the position of the second focal line. Usually, opticians try to avoid astigmatism in their optical systems. Nevertheless, it is commonly used in CD or DVD players to measure the distance between the disk and the reader head as well as for other auto-focus systems (Hsu et al. 2009). Another approach that produces an astigmatic image aberration is utilizing a tilt angle between the measurement volume and the camera, which results in off-axis imaging (Hain and Kähler 2006). This method was applied by Hain et al. (2009) to measure the velocity of particles in a macroscopic volume of $16 \times 16 \times 20 \mathrm{~mm}^{3}$. Unfortunately, the appearance of the particle images did not only change in the $z$-direction, but also in the $x y$-plane. A complex calibration procedure using a higher order polynomial fit was therefore applied by scanning light sheets with known positions. A similar approach was employed by van Hinsberg et al. (2008) to measure the flow in a volume of $5.6 \times 4.5 \times 2.1 \mathrm{~mm}^{3}$. The astigmatic aberrations were produced by an optical filter plate, placed under an angle in the light path. The particle images for a certain range of axis ratios were later artificially reconstructed and the $2 \mathrm{D}$ velocity vectors were then obtained by cross-correlation methods.

Another more elegant method is the use of cylindrical lenses. Kao and Verkman (1994) applied this technique to the measurement of the position of fluorescent particles in living cells. They used a $60 \times$ oil immersion lens to image $93 \mathrm{~nm}$ fluorescent latex beads over a depth range of $4 \mu \mathrm{m}$. The implementation of this approach to a standard $\mu$ PIV system is shown in Fig. 10. The main difference is the cylindrical lens located directly in front of the camera chip. Since no mask is used, the microfluidic device does not have to be illuminated from another direction and the SNR is improved, compared with techniques that rely on masking the optics (cmp. Fig 8). On the right side of Fig. 10 the imaging of particles is schematically shown. The cylindrical lens has a curvature only in one direction and acts as a flat window in the other direction. Therefore, two distinct focal planes are produced. In the left (red dotted) schematic the cylindrical lens causes a shortening of the distance between the focal plane and the objective lens. Particles that are close to this focal plane show a sharp and small diameter in the $x$-direction $a_{x}$ and a larger defocused diameter in the $y$-direction $a_{y}$, thus producing an oblate particle image. On the right side of the schematic (blue dashed), with the cylindrical lens rotated by $90^{\circ}$, results in a focal plane further away from the objective lens. $a_{y}$ is now smaller than $a_{x}$, resulting in a prolate particle image. The particle image width and height can now be related to the depth position by a proper calibration.

Towers et al. (2006) used a diffraction grating for multi-planar imaging as well as a cylindrical lens with a large focal length of $f=2,000 \mathrm{~mm}$ and found astigmatism to have less uncertainty for depth-wise position estimation. Furthermore, since three images are used by the method applying the diffraction grating, the field of view is limited to one-third of the camera sensor and hence is less efficient. Using both techniques, an air flow with an obstacle was later investigated (Angarita-Jaimes et al. 2006). For the calibration, a fiber light source was moved to defined positions. The measurements were later performed in a volume of $28 \times 21 \times 28 \mathrm{~mm}^{3}$ and the uncertainty in depth was estimated to be $25 \mu \mathrm{m}$ for the calibration. In this

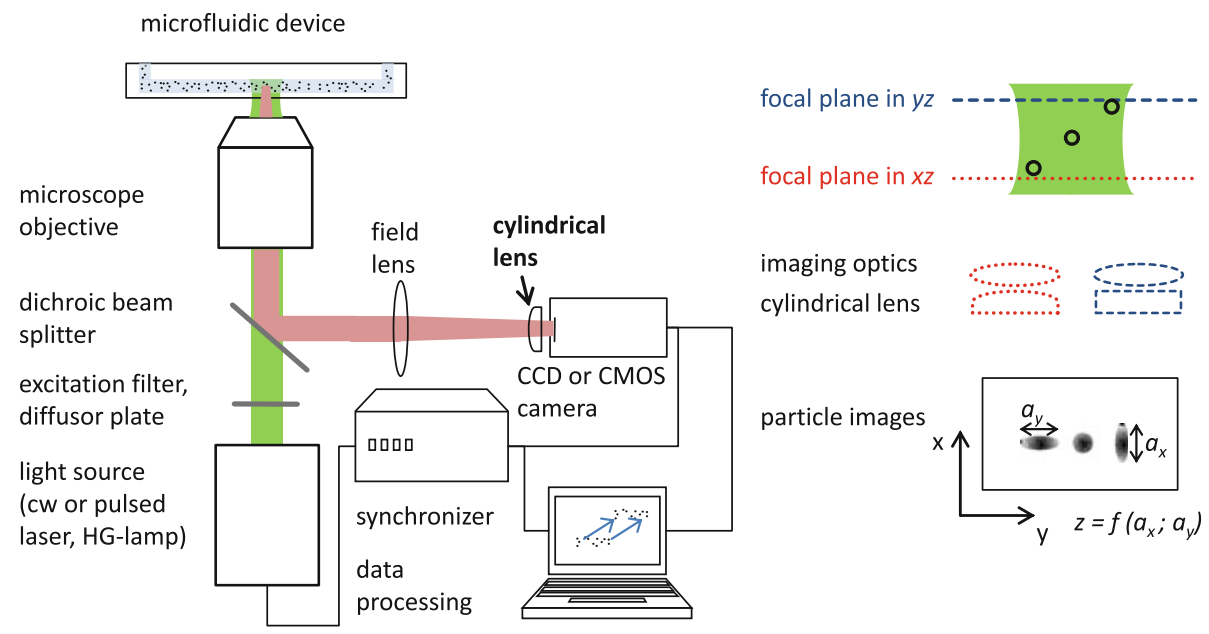

Fig. 10 Schematic of the astigmatism technique (left) and principle schematic of the imaging with reversed colors (right) 
preliminary study some 20 vectors were reconstructed. The difference of the axes of the ellipse $\left(a_{x}-a_{y}\right)$ was used for the depth-wise calibration.

Chen et al. (2009) applied the method to a of $1.8 \times 1.4 \times 0.5 \mathrm{~mm}^{3}$. They used a cylindrical lens with a focal length of $f_{\text {cyl }}=500 \mathrm{~mm}$. The distance between both focal planes was $600 \mu \mathrm{m}$. The channel with a $170 \mu \mathrm{m}$ backward facing step had a depth of $500 \mu \mathrm{m}$, ensuring that particles were not to close to either one of the focal planes. The uncertainty for the depth position was $2.8 \mu \mathrm{m}$ for the calibration images. The authors presented a theoretical estimation of the measurement depth, which is, in the first approximation $\Delta z \approx L^{2} / f$, where $L^{2}$ denotes the distance from the center of the measurement volume to the first principle plane and $f$ the focal length of the cylindrical lens. Unfortunately, no uncertainty of the single-velocity measurements was given. The rms value of the measured velocity was $3.3 \mu \mathrm{m} / \mathrm{s}$ (above one-third of $u_{\infty}$ ), even though $2.8 \mu \mathrm{m} / \mathrm{s}$ was expected from the measurement uncertainty. The experimental data were compared with numerical data and showed a good overall agreement aside from very large discrepancies at regions of high out-of-plane velocity. The images were taken in single frame mode, and the investigated Reynolds number was $R e_{h}=0.0015$. For technical applications, higher Reynolds numbers, and thus the acquisition of double frame images, are necessary (cmp. Fig. 6). Cierpka et al (2010) investigated the laminar flow in a channel with a square cross section of $500 \times 500 \mu \mathrm{m}^{2}$ at $R e=23$. The cylindrical lens used had a focal length of $f_{\text {cyl }}=150 \mathrm{~mm}$. The ratio of the particle image width and height $a_{x} / a_{y}$ was used for determining the $z$-position. This limits the measurement depth to about $40 \mu \mathrm{m}$, making scanning of volumes necessary. The data were validated against standard $\mu \mathrm{PIV}$ measurements as well as the theoretical Poiseuille flow profile and agreed within an uncertainty of $<6 \%$ of $u_{\infty}$ for a confidence intervall of 20:1. Since the measurement depth using $a_{x}-a_{y}$ or $a_{x} / a_{y}$ is limited by the two focal planes, an intrinsic calibration procedure, allowing also to use strong out-of-focus particles was developed. This procedure is based on Eq. 3 and takes into account all image aberrations since it is based on the particle images itself. Thus no scanning procedure is necessary. The same optical path as well as the same image preprocessing is used for calibration and measurements, decreasing the uncertainty of the measurements. Using this procedure, the measurement depth could be largely increased in addition. Details on the calibration as well as an experimental estimation of the measurement depth for different magnifications and focal length can be found in Cierpka et al (2011a).

Astigmatism for 3D particle imaging is already widely used. Ragan et al (2006) applied a bent dichroic mirror in the light path of a two-photon microscope to introduce astigmatism. They were able to study the motion of kidney cells expelled to external forces in real time with $28 \mathrm{~Hz}$. Quantum dots were used by Holtzer et al. (2007), observed through a cylindrical lens with a very large focal length of $f_{\text {cyl }}=10 \mathrm{~m}$, thus allowing for very precise measurements of small depths. The accuracy for the position measurements of the quantum dots was $43 \mathrm{~nm}$ for the in-plane and $130 \mathrm{~nm}$ for the out-of-plane position in a volume of $\approx 1 \mu \mathrm{m}^{3}$. Huang et al. (2008) used a 100x oil immersion lens and a EMCCD (Electron Multiplying Charge Coupled Device) to detect the position of $200 \mathrm{~nm}$ beads that were labeled with photo-switchable molecules. In a volume of $200 \times 200 \times 200 \mathrm{~nm}^{3}$ around the focal point, the accuracy was reported to be $\leq 26 \mathrm{~nm}$ for the in-plane, and $\leq 52 \mathrm{~nm}$ for the out-of-plane position. Mlodzianoski et al. (2009) compared the performance of bi-plane imaging and astigmatic imaging using a EMCCD camera. Both techniques showed a comparable measurement accuracy. For certain biological applications with thick sample sizes, the authors found the biplane imaging more adaptable but also stated that for cameras with non-negligible readout noise (like standard CCD), astigmatic imaging is favorable. Recently, time-resolved particle trajectories in an electrothermally generated micro vortex could be measured in a micro volume with $50 \mu \mathrm{m}$ in depth without traversing (Kumar et al. 2011). The three-dimensional structure of the vortex and the mechanisms of particle trapping inside the vortex were studied. The results were used to validate the numerical boundary conditions for a simulation of such a scenario. In general, the astigmatism approach based on cylindrical lenses is very easy to apply and allows for the extension of existing $2 \mathrm{D}$ measurement systems to fully $3 \mathrm{D}$ measurements without changing the illumination light path. The measurement depth and resolution can be changed by adopting the focal length of the cylindrical lens used, and the intrinsic calibration procedure (Cierpka et al. 2011a) makes the technique easily applicable without special expert knowledge. Since no mask is used, the technique is optically very efficient.

The comparison between standard $\mu \mathrm{PIV}$, stereoscopic $\mu \mathrm{PIV}$ and astigmatism $\mu \mathrm{PTV}$ showed a similar uncertainty for the in-plane velocity components (Cierpka et al. 2011b). In Fig. 11, iso-surfaces of the outof-plane component are shown for the flow over a backward-facing step. On the upper part of the figure, numerical flow simulations are shown for comparison and indicate a downward flow in the vicinity of the step. In the middle, stereoscopic measurements are presented and the region of the downward flow was well 

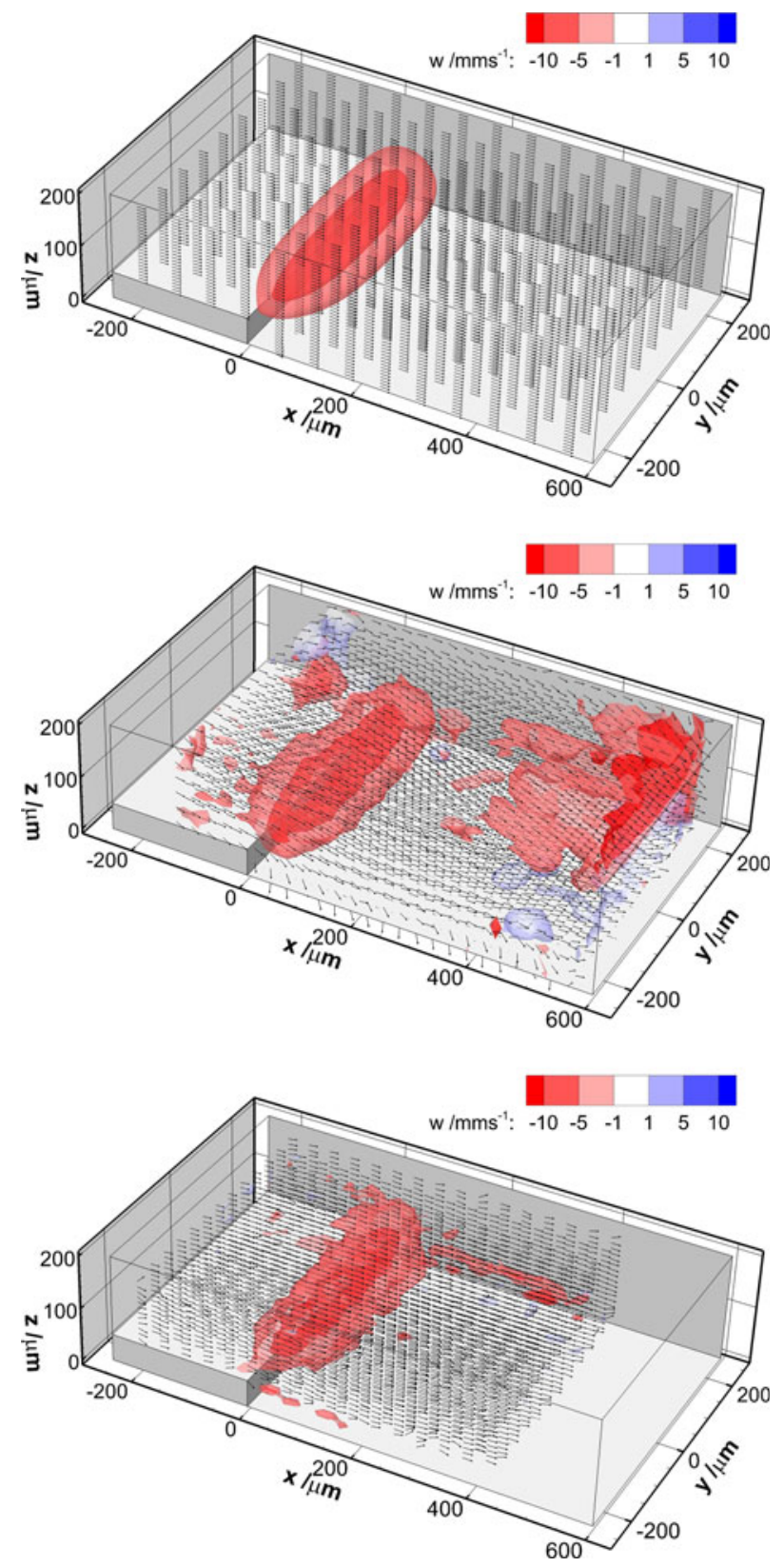

Fig. 11 Iso-surfaces of the out-of-plane velocity for the flow over a $50 \mu \mathrm{m}$ backward-facing step in a channel with a crosssection of $200 \times 500 \mu^{2}$. Numerical flow simulation (top, every 10th vector shown), stereoscopic $\mu$ PIV (middle, every 2nd vector shown) and astigmatism $\mu \mathrm{PIV}$ (bottom, every 2 nd vector shown)

captured. Although great care was taken to align the system, the focal planes of both cameras differ by several micrometers as outlined in Cierpka et al. (2011b). Thus the in-plane velocity corresponds to different regions in the volume. Since the out-of-plane components are determined from the in-plane velocity they are affected by this misalignment. The iso-surfaces downstream of the step for $x>400 \mu \mathrm{m}$ are caused by this effect and truly not physical. The results for the astigmatism particle tracking cannot be affected by a misalignment, since just one camera is used. The downward flow region close to the step is well captured, although the size of this region is slightly underestimated. However, the overall comparison showed that the uncertainty of the out-of-plane component for astigmatism $\mu \mathrm{PIV}$ is almost two times lower than for stereoscopic $\mu$ PIV (Cierpka et al. 2011b). 


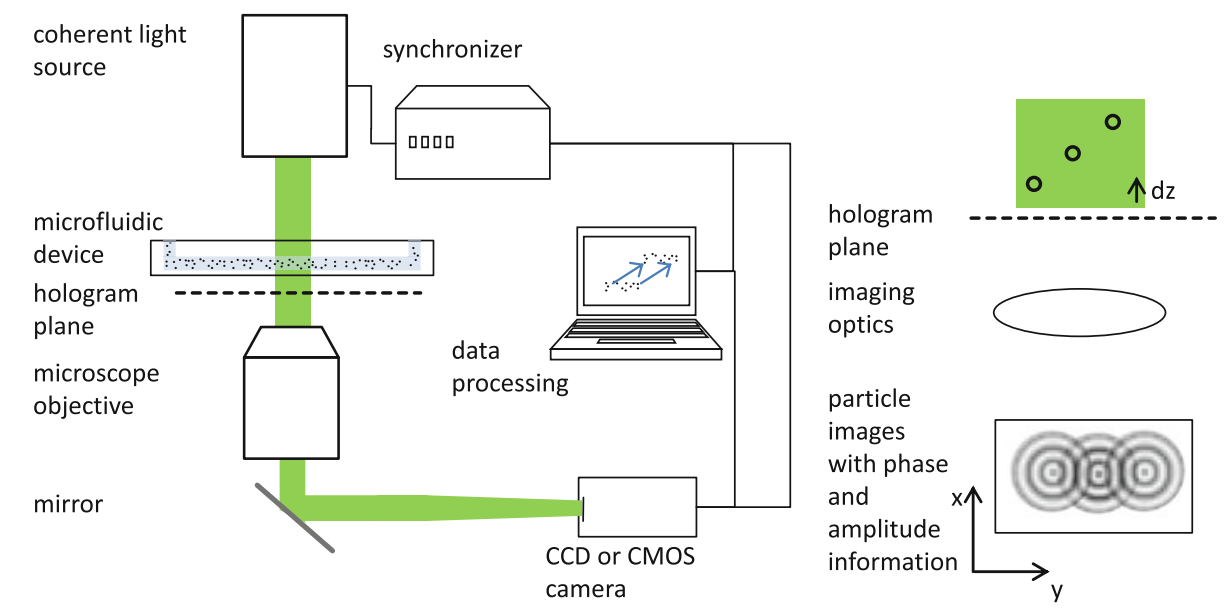

Fig. 12 Schematic of the in-line holography (left) and principle schematic of the imaging with phase and amplitude information (right)

\subsection{Digital holographic microscopy}

An alternative volumetric imaging method using a single sensor is based on holographic imaging and volumetric reconstruction, which makes additional use of the phase information of the light. Holography was invented more than 60 years ago (Gabor 1948). The word comes from the Greek language and can be translated into 'all' or 'whole' (holos-) and (grapein) 'writing'. This also reflects the property of the hologram, where each point contains information of the whole image. The hologram is the recorded interference pattern resulting from the superposition of a coherent reference wave and the object wave scattered by the object. As opposed to conventional imaging it contains amplitude and phase information. With smaller pixel size and higher sensitivity of CCD chips, a technique was developed to digitally record holograms and reconstruct the object numerically (Schnars and Jüptner 1994). A comprehensive review on digital recording configurations and numerical reconstruction techniques can be found in Schnars and Jüptner (2002). An overview of the holographic PIV technique with a detailed discussion of rising problems is given by Hinsch (2002). The configuration most often used for flow velocity measurement in microfluidic devices is the single-beam in-line holography, which is suitable since the volume of interest is nearly transparent with small opaque particles. This technique offers some advantages which are discussed in detail by Meng et al. (2004). A schematic of the configuration is shown in Fig. 12. A single laser beam is used for both the illumination and the reference wave; thus the alignment of the optics is much simpler than for dual beam and off-axis holography. The coherent laser light shines from the top onto the sample. The forward scattered light (which gives the highest signal) is interfering with the reference wave and a hologram is produced at the hologram plane. This plane can be imaged without a lens. The resolution for the in-plane position of the particles is $p_{x y} \approx \lambda / \Omega$ and for the out-of-plane position $p_{z} \approx \lambda / \Omega^{2}$ (Hinsch 2002), where $\lambda$ is the wavelength of the used light and $\Omega$ is the angular range. To have a large depth of field and thus a large measurement volume in depth direction, a low NA lens is required. Unfortunately this means a low angular range and thus the resolution decreases rapidly. Due to the low numerical aperture and the large depth of field, the reconstructed images would be highly extended in depth direction, which leads to an increased uncertainty. Furthermore, it would be difficult or impossible to image micrometer-sized particles (Katz and Sheng 2010). Several methods were proposed to increase the resolution by, e.g., using multiple viewing direction as for tomographic imaging (Soria and Atkinson 2008). However, the benefit of a single-camera arrangement, as discussed before, is lost. A compromise is the use of mirrors to record a different viewing perspective onto the same camera chip. This can be achieved by mirrors (Malkiel et al. 2003; Yamamoto and Uemura 2008) or different angles of the reference wave (Yang and Chuang 2005). One way to reduce the problem of the stretched particle images is the application of a spatial filter (Ooms et al. 2006). However, microscope objectives have large numerical apertures and thus a relatively small depth of field, which is advantageous for holography. Therefore, in digital holographic microscopy the hologram is magnified by a microscope objective, even allowing for large pixel size $(\sim 10 \mu \mathrm{m})$ high-resolution imaging. Numerical schemes must now be applied to reconstruct the object, i.e. the particle positions. However, the quality of the reconstruction strongly depends on the applied processing. First of all, the hologram suffers 
from noise (mostly by debris and imperfections of the microchannel) and has to be preprocessed. Usually, a background removal by subtracting an average field gives good results. A numerical reconstruction is then made on the basis of the clean hologram. Particle positions can now be extracted by searching points of high intensity in the reconstructed volume. Finally, PIV or PTV algorithms can be applied to estimate the velocity.

To the authors' knowledge, Coëtmellec et al. (2001) were among the first to test the idea of applying in-line holography to measure flow velocities in microchannels. Their approach was to reconstruct the intensity distribution in several slices of a channel and apply a 2D PIV algorithm to the result. They showed that the technique works in principle with particles on a glass slide and applied it later to real flow measurements (Malek et al. 2004). Malkiel et al (2003) used in-line holography with an additional prism to image a swimming copepod from two views. They were able to reconstruct the flow in a volume of $15 \times 15 \times 9$ $\mathrm{mm}^{3}$. Yang and Chuang (2005) did not have a double-shutter camera, so they used a photopolymer plate to store several successive holograms. The short time interval between the two illuminations of a double frame was accomplished by multiplexing, i.e., changing the reference wave. By switching off the object illumination, they were able to reconstruct the hologram later by scanning with a standard low-speed CCD camera. The technique was applied to a straight channel flow and a backward-facing step and showed a deviation of 9\% in comparison with standard $\mu$ PIV. Satake et al. (2004) used in-line holography without any imaging lens to measure the transient flow around an obstacle in a volume of $4 \mathrm{~mm}^{3}$, with an out-of-plane resolution of $20 \mu \mathrm{m}$. They reported an error of 5\% for the mean in-plane velocity. A similar setup, consisting of a Nikon $40 \times(\mathrm{NA}=0.55)$ magnification lens was used by the same authors in 2005 (Satake et al. 2005) to enhance the spatial resolution. They measured the flow field in a circular pipe over a depth of $40 \mu \mathrm{m}$ with an out-of-plane resolution of $0.2 \mu \mathrm{m}$. Later, they were able to increase the measurement depth to $70 \mu \mathrm{m}$, close to their original objective of $100 \mu \mathrm{m}$ (Satake et al. 2006). They used a high-speed CMOS camera with a relatively large pixel size of $16 \mu \mathrm{m}$ and 40x magnification. Velocity profiles showed a good agreement with theoretical results, although the determination of the depth position from the middle of the channel seemed to be underestimated. Lee and Kim (2007) applied in-line holography for the measurement of the velocity profile in a straight channel. They obtained a measurement depth of $100 \mu \mathrm{m}$ using a 60x magnification lens with a highspeed CMOS camera $(17 \mu \mathrm{m}$ pixel size) and $2 \mu \mathrm{m}$ particles to seed the flow. For different seeding densities, they showed that the error decreases with increasing seeding concentration. However, the number of reconstructed particles also strongly decreases with increasing seeding concentration. In a later work, Lee and Kim (2009b) successfully applied the system to measure the secondary flow in curved microchannels with a diameter up to $300 \mu \mathrm{m}$. Ooms et al. (2009) applied holographic PTV with $2 \mu \mathrm{m}$ particles to the flow in a T-shaped mixer. They made a thorough analysis of all the parameters for setting up a holographic microscopy experiment. They were able to measure the flow inside a volume of $1 \times 1 \times 1 \mathrm{~mm}^{3}$ in a T-mixer. Timeresolved measurements were obtained using a high-speed camera. A PTV algorithm was applied and $\sim 18,000$ vectors were finally reconstructed. The investigation successfully showed the application of hologrametric methods to micro flows, although the data processing was time consuming (several days for 50 holograms at that time). Recently, Choi and Lee (2009) used a cinematographic version of digital holographic microscopy to study the motion of red blood cells in microtubes of 100 and $350 \mu \mathrm{m}$ in diameter. The authors tested different focus functions for the reconstruction of particle positions and found that the squared Laplacian function yields the smallest depth of focus $(7.4 \mu \mathrm{m})$ and thus the lowest rms position error which is $2.3 \mu \mathrm{m}$ in depth-wise direction. They reported an uncertainty of around $9 \%$ of the mean velocity in the tube for the in-plane velocity and 33\% for the out-of-plane. Since the volumetric position of particles can be reconstructed in the whole channel, holographic microscopy was used by the same authors to study the migration of larger particles in a pipe flow (Choi and Lee 2010). Without going too much into detail, the probability density function of the particle distribution was measured and the formation of the so-called Segré-Silbergerg annulus was studied for varying Reynolds numbers and particle diameters.

Aside from the measurement of particles positions in liquids, microscopic holography could also be used to measure the size and velocity of droplets in a volume. Nguyen et al. (2010b) used a magnification of $4 \times$ to measure the evaporation of diesel fuel. However, due to the comparably low numerical aperture, the droplets showed a diameter of $\approx 1 \mathrm{~mm}$ in the axial direction, which corresponds to $15 \times$ the in-plane diameter. Another interesting use of holography for microfluidic measurements was presented by Di Leonardo et al. (2006). The authors used a hologram to trap particles at certain locations in the volume by a spatial light modulator (SLM). When the particles were released, they were assumed to follow the flow and their velocity was measured by particle tracking. Since the position in the depth direction can be controlled precisely, no errors due to DOC occur, even without reconstructing the particle position from holograms. 
Whereas the defocusing methods described in Sects. 3.2, 3.3 and 3.4 rely on spherical particles, holography allows for the reconstruction of non-spherical particles, such as, e.g., micro organisms. For a given optical setup, the depth of focus is proportional to $d^{2} / \lambda$ (Meng et al. 2004), where $d$ is the particle size and $\lambda$ the wave length of the coherent light. Unfortunately, the micro organisms are often rather large $d>30 \mu \mathrm{m}$ and thus the depth-of-focus reaches values of up to $1 \ldots 3 \mathrm{~mm}$. Due to the large depth-of-focus, special numerical focus functions have to be used to exactly determine the depth position of the bio-sample. Lee et al. (2011) reported the best results for a focus function that relies on the variation in the axial intensity gradient. They reported an rms uncertainty in the axial position of $21.5 \mu \mathrm{m}$ for $30 \mu \mathrm{m}$ particles. Using a high-speed camera, they were able to reconstructs the trajectories of differently shaped microorganisms (dinoflagellates).

In general, holography shows a great potential, especially for microscopic flows. The reconstruction provides the fully three-dimensional information of the particle position. By the magnification of the holograms with microscope objectives, the resolution can be greatly increased compared with lensless holography. The field of view will be limited but is still sufficiently large for microfluidic devices. However, a large drawback is the numerically costly reconstruction process of the holograms and their sensitivity to noise. The problem of the time-consuming reconstruction will disappear with more effective algorithms and the use of GPUs. In order to solve the second issue and to increase the SNR of the holograms, in-line holography (forward scattering) is used. Unfortunately, this limits the technique to very low seeding densities to avoid a lack in the data. Another problem is the reduced resolution in the depth direction. Reconstructed particle images are often elongated in the depth direction (up to 10 times Hinsch (2002)), thus lowering the accuracy.

\section{Tracking or correlation}

In principle all the different imaging techniques are suitable to determine the $2 \mathrm{D}$ or $3 \mathrm{D}$ position of the tracer particles in the flow for certain experimental conditions. Despite their differences, the next step is the estimation of the velocity field from the displacement of the particle images. In the early days of PIV, the image quality was very low due to low laser power and less sensitive cameras. However, with correlationbased methods the particle image displacement could be estimated quite robustly compared with particle tracking techniques, which are very sensitive to noise (Stanislas et al. 2003). Another advantage is that the data are sampled on a regular grid, which allows for the direct calculation of derivatives and simplifies the visual analysis of the flow topology, the identification of flow structures and the reliable detection of outliers. Lately, the validation of high spatial resolved results from computational fluid dynamics is strongly desired. PIV methods are due to the spatial averaging and the DOC limited in this respect and particle tracking algorithms come into focus again.

Correlation-based methods are mostly used for macroscopic flows. The seeding concentration is usually not a problem in these cases. Additionally, gradients are often not so strong and the assumption for PIV that all particles belonging to a correlation window move with the same velocity holds true. However, the camera sensitivity and the quality of the fluorescence coating for tracer particles have been improved much in the recent years. The development of very stable and cheap diode-pumped lasers with longer pulse duration than the regularly used Nd:YAG double-pulse lasers also increased the image quality for fluorescent particles considerably. However, a tracking algorithm based on high-quality images is quite accurate. From theoretical assumptions a tracking procedure also seems favorable to a correlation-based velocity evaluation for microscopic flows for several reasons:

- In microfluidics, the particles are typically imaged with larger diameter than in macroscopic PIV. The resolution for correlation methods depends on the particle image diameter and is limited to $2 \times$ the particle image diameter (Kähler and Scharnowski, 2011).

- Individual particles can easily be identified and their position can be determined with sub-pixel accuracy.

- The seeding concentration is often low, thus limiting the minimum size of the interrogation windows. Hence, strong in-plane gradients at solid walls or in shear regions are underestimated.

Furthermore, out-of-focus particles bias the measurements due to their contribution to the correlation function (Rossi et al. 2011). If the 3D position of a particle is known, or in-focus particles are used for the velocity estimation, these effects can be greatly reduced and the accuracy can be increased. 
Different authors directly compared tracking algorithms to correlation-based data processing on the same dataset. Yu et al (2009) studied stereoscopic PIV and PTV with different microscopes. They used immobilized particles in an epoxy target and a micro jet flow to compare particle tracking and PIV via cross correlation. The results show an increased uncertainty using correlation-based algorithms, especially for low numerical aperture objectives. For small out-of-focus displacement the uncertainty for the correlation-based algorithm was up to five times larger than for tracking. For the jet measurements, the PIV algorithm underestimates the jet core velocity by $\approx 8 \%$. Furthermore, the vector spacing using correlation was $47.4 \mu \mathrm{m}$ compared with a mean distance of the vectors of $26.2 \mu \mathrm{m}$ for PTV. Bown et al. (2006) reported for a stereoscopic setup, a much lower uncertainty of $2 \%$ of the mean velocity for the in-plane component and $3 \%$ for the out-of-plane component using a super-resolution algorithm with particle tracking compared with $3 \%$ for the in-plane component and $7 \%$ for the out-of-plane velocity using correlation solely. Furthermore, they could measure the 3D position of particles and thus increase the depth resolution. To evaluate the drawbacks of different methods and determine weather pre-processing could overcome the limitations due to DOC, a simple test experiment in a straight channel was performed. The channel was made from elastomeric polydimethylsiloxane (PDMS) on a $0.6 \mathrm{~mm}$ thick glass plate by the Institute for Microtechnology of the Technical University Braunschweig and had a cross section of $510 \times 205.5 \mu \mathrm{m}^{2}$. Particles that stuck to the top and bottom wall were used to measure the channel height. These particles were imaged while scanning in the $z$-direction with a step size of $0.5 \mu \mathrm{m}$. The channel height was determined using a fit of the rms intensity as focus function (Sun et al. 2004). The constant flow rate was generated by pushing distilled water at a rate of $20 \mathrm{ml} / \mathrm{h}$ through the channel using a high-precision neMESYS syringe pump $($ Cetoni $\mathrm{GmbH})$. The flow in the channel was homogeneously seeded with polystyrene latex particles with a diameter of $2 \mu \mathrm{m}$ (Microparticles $\mathrm{GmbH}$ ). The particle material was pre-mixed with a fluorescent dye and the surface was later PEG modified to make them hydrophilic. Agglomeration of particles at the channel walls can be avoided by this procedure, allowing for long-duration measurements without cleaning the channels or clogging. For the illumination, a two-cavity frequency-doubled Litron Nano S Nd:YAG laser system was used. The laser was coupled with the inverted microscope (Zeiss Axio Observer) by a fiber optic. The image recording was performed at a magnification of 20x (Zeiss LD Plan-Neofluar, NA $=0.4$ ) with the DaVis 7.4 software package from LaVision $\mathrm{GmbH}$. The images were acquired by a 12-bit, $1,376 \times 1,040$ pixel $^{2}$, interline transfer CCD camera (PCO Sensicam QE) in double-exposure mode. The time delay between the two successive frames was set to $\Delta t=100 \mu$ s. 8,000 images were recorded at $z=93 \mu \mathrm{m}$ with an intermediate seeding concentration of $5 \times 10^{-5}$ in focus particles per pixel which corresponds to $0.4 \%$ of sensor area covered by particle images or approximately 70 particles per frame. The mean in-focus particle image diameter was around 10 pixels.

For the estimation of the velocity, the same part of the image was used for the different techniques. In Table 1, the parameters for the different processing methods are listed. Special considerations are tabulated such as the use of raw images (raw) or preprocessed images (pre). The preprocessing consists of simply subtracting a local minimum within a kernel size of 4 pixels to remove the background intensity and a constant intensity subtraction of 20 counts to filter the out-of-focus particles left by the background removal. The cross correlation was performed with adaptive window shifting and decreasing interrogation window size starting from $128 \times 128$ pixels to $64 \times 64$ pixels with $50 \%$ overlap, using DAVIS 7.4 by LaVision $\mathrm{GmbH}$. An outlier detection (Westerweel and Scarano 2005) was used to remove strong outliers. Averaging of the vector fields yields to the final velocity profiles indicated with PIV (raw) and PIV (pre). The averaging or summation of the correlation maps is an effective technique to deal with low seeding concentrations and low signal-to-noise ratios, especially for the processing of PIV data (Meinhart et al. 2000). Currently, hybrid methods are also discussed by Nguyen et al. (2010a) and Samarage et al. (2011). However, here we present data of pure correlation averaging indicated with sum corr $\left(\operatorname{raw}_{a ; b}\right)$ and sum corr (pre). Since the correlation

Table 1 Main parameters for the different processing methods

\begin{tabular}{ll}
\hline Abbreviation & Description \\
\hline PIV (raw) & Raw images, $64 \times 64$ pixels with $50 \%$ overlap, vector averaging \\
PIV (pre) & Preprocessed images, $64 \times 64$ pixels with 50\% overlap, vector averaging \\
Sum corr $\left(\mathrm{raw}_{a}\right)$ & Raw images, norm. correlation, $64 \times 64$ pixels with 50\% overlap, correlation averaging \\
Sum corr $\left(\mathrm{raw}_{b}\right)$ & Raw images, not norm. correlation, $64 \times 64$ pixels with 50\% overlap, correlation averaging \\
Sum corr $($ pre) & Preprocessed images, norm. correlation, $64 \times 64$ pixels with $50 \%$ overlap, correlation averaging \\
Single pixel & Raw images, single pixel correlation, correlation averaging over 40 pixels \\
PTV (raw) & Raw images, $128 \times 128$ pixels cross correlation and final PTV step \\
PTV (pre) & Preprocessed images, $128 \times 128$ pixels cross correlation and final PTV step \\
\hline
\end{tabular}


peak height strongly depends on the intensity and the number of the particles in the current interrogation volume (Westerweel 1997), the correlation plane is often normalized by its variance. This yields to a peak height between 0 and 1 and can be used to check the correlation's quality and to disregard outliers (Tropea et al. 2007). Also, the bias due to varying signal content and background intensities over the image are successfully suppressed by this procedure. A comparative analysis of the sum of the standard correlation planes and the sum of the "normalized-by-variance" correlation planes is presented to emphasize the great difference on the final results. For sum corr $\left(\mathrm{raw}_{a}\right)$, the single-correlation planes were normalized prior to averaging and for sum corr $\left(\operatorname{raw}_{b}\right)$ no normalization was done. A method to greatly increase the spatial resolution is the single-pixel ensemble correlation, is described by (Westerweel et al. 2004; Kähler et al. 2006). A signal is reconstructed for up to every pixel, containing all image information over the ensemble (in this case all 8,000 images). This signal is then correlated with signals of neighboring pixels and a displacement can be estimated for each pixel. The method applied here is described in greater detail in Scharnowski et al. (2011) and referred to as single pixel in the table. The last method used is a so-called super-resolution algorithm (Keane et al. 1995). Particle tracking is performed as the final step of the analysis with DAVIS 7.4 by LaVision GmbH. A search region predictor for the tracking is based on a cross correlation on a larger grid $(128 \times 128$ pixels in this case $)$. The accuracy for low seeding concentrations is not affected by the predictor step, but it improves the tracking performance in higher seeding concentrations.

In Fig. 13 the normalized velocity profiles are shown for the different techniques. The results on the left side are always based on the raw images, also including out-of-focus particles. On the right side the preprocessed images were used and mainly in-focus particles contribute to the results. The whole velocity
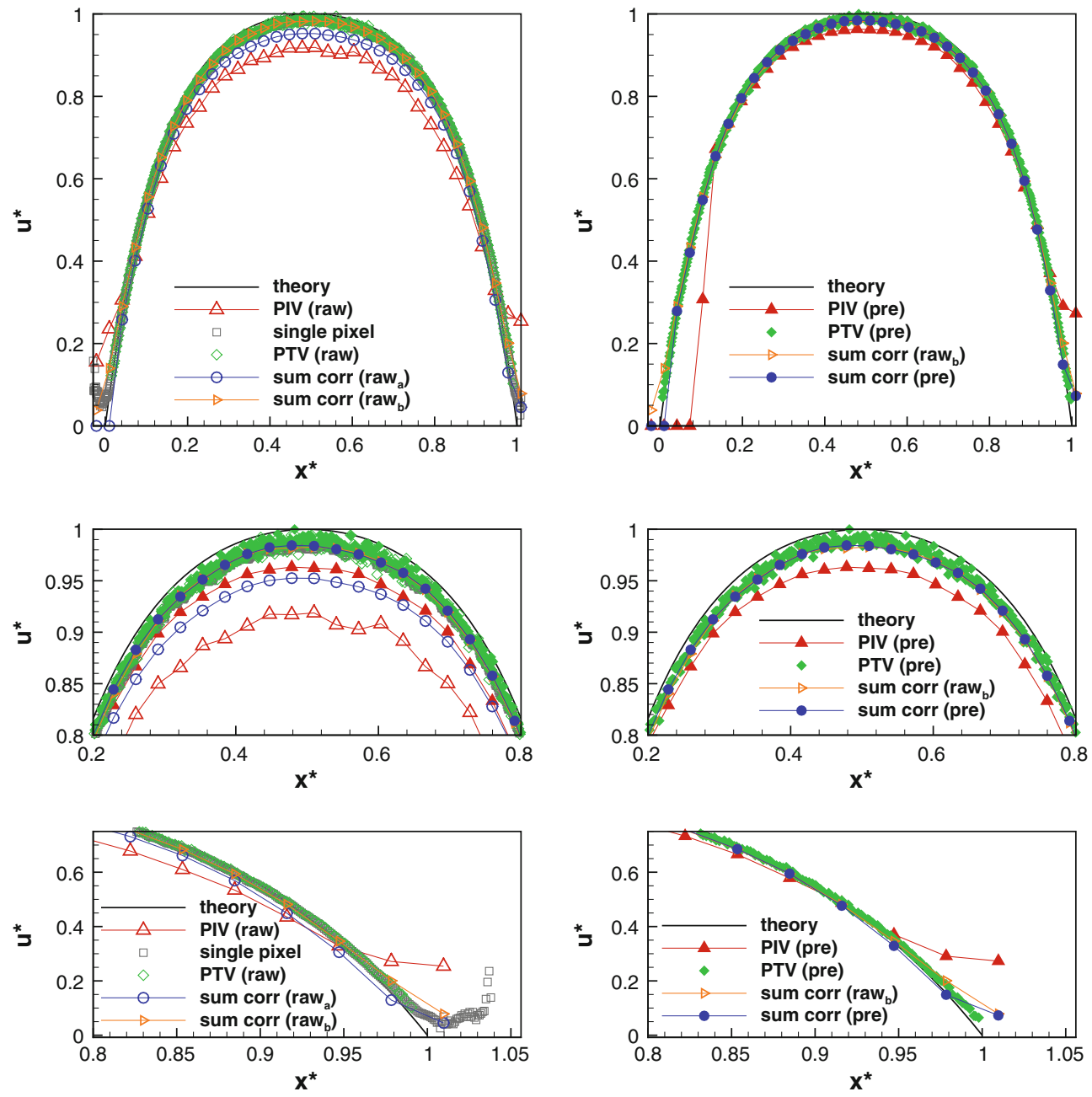

Fig. 13 Velocity profiles at $z=93 \mu \mathrm{m}$ for the different techniques based on the raw images (left) and preprocessed images (right). Whole profile (top), center part (middle) and close to the wall (bottom) 
profiles can be seen in the upper part of Fig. 13. Results obtained by all techniques resemble the expected parabolic velocity profile, but their respective errors vary substantially. The theoretical profile, based on the volume flow rate supplied by the syringe pump and the measured channel cross section is drawn in all diagrams for comparison.

Especially for correlation-based methods, it is known that the velocity is affected by two averaging errors. The first one is the DOC and is caused by out-of-focus particles that contribute to the correlation and might move with a different velocity than in-focus particles, as already described in Sect. 1.1. This underestimation can be clearly seen in the vector averaging profiles (middle right part of Fig. 13) calculated from the raw images, where the error at $x^{*}=0.5$ is up to $9 \%$ of $u_{\max }^{*}$. Using the correlation averaging, the error decreases to $5 \%$ of $u_{\max }^{*}$ for the use of the averaged normalized correlation functions. Although the same images are used, correlation averaging always performs better than vector averaging for low seeding concentrations. Using the non-normalized correlation planes for the averaging shows better results than using normalized correlation planes. These biases can be explained by the different weighting of out-offocus particles' contribution.

For the vector averaging, a valid vector, based on a single two-frame correlation is determined even if only out-of-focus particles are present. Using vector averaging, this erroneous vector has the same weight as all other vectors in the ensemble and causes a bias in the final result. Also, outliers in the data bias the averaged result towards zero, since it is assumed that outliers are randomly distributed with a zero mean (Westerweel et al. 2004; Samarage et al. 2011). Finally, the velocity is strongly underestimated. On the other hand, if correlation planes of interrogation windows, containing only out-of-focus particles and/or noise, are normalized before the correlation average is calculated, they get the same weight as all other valid correlation maps and thus smearing the final averaged correlation peak. The influence by out-of-focus particles is thus very pronounced and the velocity is strongly underestimated. If the correlation planes are not normalized, the correlation of in-focus particle images (with higher intensities) will result in a much higher correlation peak height than the correlation of low-intensity out-of-focus particles. Due to this weighting, the influence of out-of-focus particles is decreased automatically without image preprocessing. As can be seen in Fig. 13, the error in the center of the channel is only about $1.5 \%$ of $u_{\max }^{*}$. The effect of the normalization is further illustrated in Fig. 14, where the summation of all 8,000 correlation planes for one interrogation window is shown. The graphs show the profiles through the correlation planes in both the $i$ - and $j$-directions and are divided by their maximal values for better visibility.

The correlation peak in this representation is centered at $i=15 ; j=16$ and the position with sub-pixel accuracy is given in the top right corner. It is clear that the peak for both averaging methods is smooth. However, even though the correlation plane is centered at certain pixel values, the sub-pixel peak determination by a Gaussian peak fit results in slightly different values as indicated in the figure. Furthermore, it is also obvious that the summation of not normalized correlation maps results in an averaged correlation map that looks quite Gaussian with a much larger signal-to-noise ratio. Again, this effect is due to the low absolute height of the correlation coefficient and thus lower influence of out-of-focus particles.

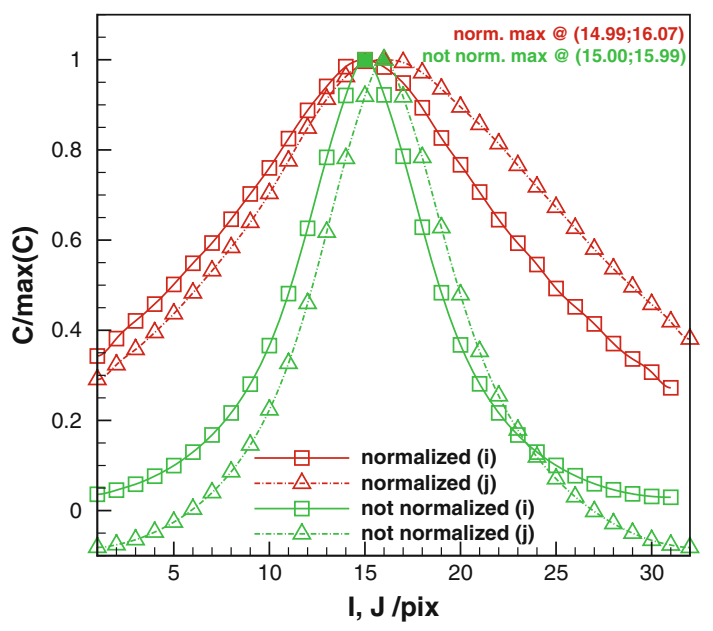

Fig. 14 Profiles of the summation of the 8,000 successive correlation planes in the $i$ - and $j$-direction, with and without normalization of the individual correlation planes 
Another way of reducing the effect of out-of-focus particles is to use image preprocessing. Using this method, the error decreases to $4 \%$ of $u_{\max }^{*}$ at $x^{*}=0.5$ for vector averaging and $2 \% u_{\max }^{*}$ for correlation averaging. However, due to the image preprocessing the signal content for the correlation is also lowered. This causes the problem that for some image pairs no valid vectors were found, especially at the left side of the channel, as can be seen at the upper right plot in Fig. 13. Another inherent problem that does not depend on the preprocessing arises at the side walls for the cross-correlation methods, where strong gradients are present. Due to the finite window size, the estimated velocity is an in-plane average over the window and gradients are always underestimated. This can be clearly seen in the lower part of Fig. 13. Approaching the side walls, the gradients are steeper and steeper and, additionally, no particles with zero velocity are present outside of the channel. Thus, the measurements are averaged and biased towards higher velocities. A good agreement with the theoretical values can be found up to a distance of 5\% of $x^{*}$ from each wall. This corresponds to approximately half the interrogation window size.

The velocity profile for the single-pixel evaluation is almost not effected by the DOC since in-focus particles contribute much more to the correlation. The error in the center at $x^{*}=0.5$ is just $1.0 \%$ of $u_{\max }^{*}$. The resolution is much better than for the other correlation-based methods. Since the single-pixel evaluation minimizes the effect of spatial in-plane averaging, the gradients at the side walls are quite well predicted. Using correlation averaging without normalization and decreasing the interrogation window size to $1 \times 1$ pixel would finally yield the same results. Interestingly, the velocity close to the side wall starts to deviate from the theoretical solution. This effect is caused by the finite size of the particle images. The limit for the resolution is given by the diameter of the particle image. The mean size of the images for in-focus particles was around 10 pixel, which corresponds for the current setting to $\approx 1 \%$ of $x^{*}$. Since no particles with zero velocity are present on the other side of the wall, the result is biased towards higher velocities. The first unbiased vector can be expected at $1.86 \times$ the particle image diameter from the wall (Kähler and Scharnowski 2011). However, even for large particle image diameters this effect is small compared with the averaging effect of the interrogation windows and results in a relative overprediction of the velocity (based on the local theoretical velocity at the current position) of $<8 \%$ of $u^{*}$.

Since a threshold is set for the PTV approach, on weather a particle image is valid or not, only focused particles are taken into account and the results for raw and preprocessed images do not differ significantly. The underestimation in the center of the channel at $x^{*}=0.5$ is just $1 \%$ of $u_{\max }^{*}$. For the profiles presented here, a simple binning to integer values of pixel was done for the position in $x$-direction when averaging the PTV data. Thus, the resolution is the same as for the single-pixel evaluation. However, since the mean distance between the vectors is decreasing with increasing measurement time, the resolution can be greatly enhanced, even to sub-pixel position for steady or periodic flows. For correlation-based methods, the spatial resolution is fixed and cannot be increased for a greater amount of images. Recently, a local universal outlier detection algorithm for PTV data was proposed by Duncan et al. (2010). The outlier detection is based on the normalized residuum, taking the surrounding values weighted by their distance into account. However, for the data presented here, an outlier removal was not necessary. Nevertheless, it has to be stated that the uncertainty of a single PTV measurement is higher than for the correlation-based methods, where six to ten particle images contribute to one vector estimation. If six to ten PTV data points are averaged, the uncertainty usually decreases below the value for correlation-based methods (Sato et al. 2003; Cierpka et al. 2011b). Another advantage of particle tracking methods over correlation-based methods arises regarding instationary or pulsatile flows. Poelma and Westerweel (2010) discussed the problem of measuring reliable velocity averages for non-stationary flows using the sum of correlation. Since the correlation planes for flow fields with different velocities are averaged, the pdf of the velocity is hidden in the averaged correlation and the highest peak might not give the real mean velocity. If the particle images show good quality, a PTV algorithm can be used which provide the full pdf of the velocity and the real mean velocity can be estimated.

Nevertheless, although the uncertainty for single measurements is higher in PTV than in PIV, tracking methods have some benefits:

- The measurements are not biased by the spatial averaging due to depth-of-correlation.

- No in-plane spatial averaging is smoothing the gradients close to walls or in regions of high shear.

- For steady flows, the average distance between vectors can be decreased by taking more images and thus the spatial resolution can be increased.

Particle images in microscopy are often of higher quality than for macroscopic imaging and the seeding concentration (in particle images per pixel) is usually low. Thus tracking techniques seem to have a higher potential when dealing with steady or periodic flows and when averaging over a longer time period is 


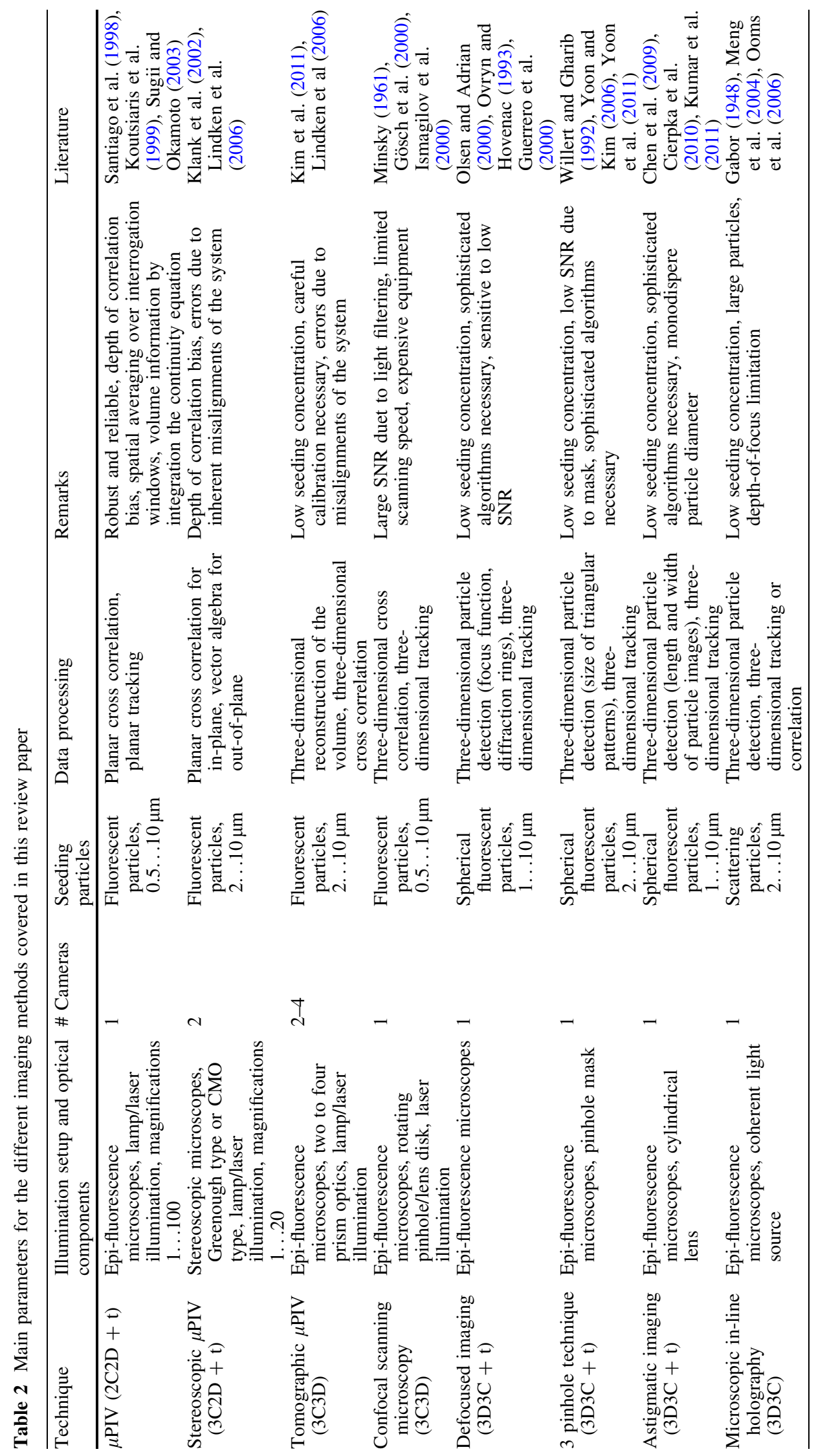


possible. If the instantaneous velocity field is needed and the experimental conditions only allow for a very low SNR, a robust method such as cross correlation, which makes use of larger interrogation window sizes, is the technique of choice.

\section{Conclusion}

This paper aimed at providing an overview of recent developments and help to choose a suitable optical velocity measurement technique for microfluidic applications. Since the future applications will mostly feature three-dimensional flows, the focus was on techniques able to provide all three components (3C) of the velocity vector in the measurement volume (3D). The main parameters of all different techniques are summarized in Table 2. First the standard epi-fluorescence $\mu$ PIV was introduced as the state-of-the-art technique. Using this technique, it is possible to resolve two components of the velocity vector in the measurement plane. Using a high-speed camera, the technique can provide time-resolved data $(2 \mathrm{D} 2 \mathrm{C}+\mathrm{t})$. Since a lot of recent microfluidic applications show very complex and fully three-dimensional velocity fields, the availability of reliable 3D techniques is mandatory. Integrating the continuity equation can extend the measurements to the third component for stationary flows. Special care has to be taken at the boundaries, since the uncertainty will strongly affect the results. Another inherent problem is the bias of the results due to out-of-focus particles, the so-called depth of correlation bias.

A way to extend a system to the third dimension is the use of multiple perspectives. Therefore, multicamera approaches (stereoscopic and tomographic $\mu \mathrm{PIV}$ ) were discussed. These approaches were successfully applied by different researchers. However, the main difficulty of these approaches is the complex calibration. Small errors due to slight camera misalignments will cause a large measurement uncertainty. In addition, the accuracy is limited by the small viewing angles of the two or more perspectives. This drawback can be overcome, to a certain extent, with sophisticated optical alignments. Nevertheless, single-camera methods with particle depth coding have the great advantage that they do not include complicated calibration procedures to perform full $3 \mathrm{D}$ measurements with a single optical access.

Confocal scanning microscopy can be used to greatly enhance the SNR by filtering light that is coming from other areas than the focal spot with a pinhole. Just one optical access is necessary and the full 3D particle position information can be measured in the whole volume. This makes a fast scanning of the whole volume necessary and thus the scanning speed currently limits temporal resolution, plus the equipment is very expensive. Nevertheless, the particle distribution in the whole volume can be reconstructed without bias due to DOC.

Imaging based on defocus or aberrations, as well as holographic microscopy, does not suffer from the DOC problem either. All these techniques are real three-dimensional measurement approaches. Using highspeed cameras, all techniques can be used for time-resolved sampling of the flow. Measuring the size of the diffraction rings, or the defocused particle images, is a straight forward way to extend the system to the third dimension without scanning or multiple perspectives using standard equipment. For these techniques, different calibration functions are discussed in the literature. However, for some optics, the defocus function is not unambiguous, thus limiting the measurement depth. Breaking the axis symmetry of the imaging system helps to overcome the ambiguity. One way is to use a mask. Three-pinhole masks were widely used in the past resulting in a triplet of particle images for one particle in the flow. The size of this triplet can be related to the depth position of the particles. The three-pinhole technique is very easy to apply and makes use of standard $\mu$ PIV equipment. By introducing a pinhole mask in the optics, the quality of the imaging lens is not affected and no additional image aberrations occur. The measurement depth can be easily adopted and the technique is widely applicable. A drawback is that the laser illumination has to come from the top and thus a second optical access and a transparent sample is necessary. Furthermore, the strong filtering of light by the pinhole mask lowers the SNR. The detection of corresponding images of a triplet is another challenge that requires sophisticated image processing and low seeding concentrations. The astigmatic imaging technique uses a cylindrical lens to break the axis symmetry of the imaging system. The particle images appear as ellipses according to their depth in the volume. Using a cylindrical lens is a low-cost and easily applicable method that enables fully 3D3C measurement with standard microscopic equipment. The influence of unwanted image aberrations can be excluded by a calibration. In comparison with the threepinhole technique, no second optical access is necessary and the SNR is much higher since all of the light is used. Recently, the technique was demonstrated to show a lower uncertainty compared with stereoscopic imaging. All these techniques can be used for fully 3D3C velocity and particle distribution measurements. However, to use the full depth of the imaged volume, astigmatic imaging requires particles featuring a very 
narrow monodisperse distribution of the diameter. Usually such particles can easily be produced and purchased; however if not available, the reliable measurement region is limited to be within both focal planes.

An approach that makes use of the phase information of the scattered light wave is holographic microscopy. Digital holographic microscopy provides the full 3D positions of particles in the whole volume and is already widely used. The technique is not limited to spherical particles and thus is also suited to measure the motion of micro organisms. However, the technique is quite costly in terms of computer time for the reconstruction. Furthermore, for in-line holography, coherent light from the cameras' opposite direction has to be used; thus a second optical access is necessary and the particle concentration is limited. Another problem is the out-of-plane accuracy. Even high NA systems produce particle images that have a reconstructed extension in depth direction of 2-10 in-plane diameters (Katz and Sheng 2010).

Once a suitable imaging technique is chosen, the question arises weather correlation-based or tracking methods should be used for the velocity estimation. In general, it is the belief of the authors that particle tracking will gain greater attention for future applications in microfluidics. One of the main reasons is that 3D tracking schemes especially do not suffer from a measurement bias due to DOC. Typically, high in-plane gradients are also present in microfluidic devices. Since no spatial averaging for an interrogation window is necessary, tracking is the better suited method. Usually the seeding concentration in microfluidics is quite low compared with macroscopic measurements. Methods to improve correlation-based schemes, like correlation averaging, image averaging or hybrids of both are proposed (Nguyen et al. 2010a). However, the particle images themselves are of good quality due to more sensitive cameras, higher quality fluorescent dyes and improvements in the particle production. Thus, the processing of single-particle images reaches the same quality compared with correlation. Whereas for correlation-based methods one velocity vector is estimated by the displacement of six to ten particle images, a data point is achieved for each particle image in tracking. In addition, if the particle positions are known, this information can be used, for example, to reconstruct interfaces between different streams (Rossi et al. 2011a) which seems to have a large potential to analyze mixing phenomena in microfluidics. Using particles with a dye that shows a certain sensitivity, e.g., to temperature, $\mathrm{pH}$-value or even pressure, it is possible to extend the techniques to measure the threedimensional velocity vector in the volume and the distribution of a scalar in the same volume simultaneously.

Acknowledgments Financial support from Deutsche Forschungsgemeinschaft (DFG) and fruitful discussions with Prof. Okamoto are gratefully acknowledged. The authors would also like to thank Stefanie Demming and the Institute for Microtechnology at the Technische Universität Braunschweig and Rodrigo Segura from the Bundeswehr University Munich for their kind support which is gratefully appreciated.

\section{References}

Adrian RJ (1997) Dynamic ranges of velocity and spatial resolution of particle image velocimetry. Meas Sci Technol 12:1393. doi:10.1088/0957-0233/8/12/003

Angarita-Jaimes N, McGhee E, Chennaoui M, Campbell HI, Zhang S, Towers CE, Greenaway AH, Towers DP (2006) Wavefront sensing for single view three-component three-dimensional flow velocimetry. Exp Fluids 41:881-891. doi: 10.1007/s00348-009-0737-Z

Angele KP, Suzuki Y, Miwa J, Kasagi N (2006) Development of a high-speed scanning micro PIV system using a rotating disc. Meas Sci Technol 17:1639-1646. doi:10.1088/0957-0233/17/7/001

Arroyo MP, Greated CA (1991) Stereoscopic particle image velocimetry. Meas Sci Technol 2:1181-1186. doi: $10.1088 / 0957-0233 / 2 / 12 / 012$

Atkinson C, Soria J (2009) An efficient simultaneous reconstruction technique for tomographic particle image velocimetry. Exp Fluids 47:553-568. doi:10.1007/s00348-009-0728-0

Bourdon CJ, Olsen MG, Gorby AD (2004) Validation of an analytical solution for depth of correlation in microscopic particle image velocimetry. Meas Sci Technol 15:318-327. doi:10.1088/0957-0233/15/2/002

Bown MR, MacInnes JM, Allen RWK (2005) Micro-PIV measurements and simulation in complex microchannel geometries. Meas Sci Technol 16:619-626. doi:10.1088/0957-233/16/3/002

Bown MR, MacInnes JM, Allen RWK, Zimmerman WBJ (2006) Three-dimensional, three-component velocity measurements using stereoscopic micro-PIV and PTV. Meas Sci Technol 17:2175-2185. doi:10.1088/0957-0233/17/8/017

Bown MR, MacInnes JM, Allen RWK (2007) Three-component micro-PIV using the continuity equation and a comparison of the performance with that of stereoscopic measurements. Exp Fluids 42:197-205. doi:10.1007/s00348-006-0229-3

Brücker C (1995) Digital-particle-image-velocimetry (DPIV) in a scanning light-sheet: 3-d starting flow around a short cylinder. Exp Fluids 19:255-263. doi:10.1007/BF00196474

Brücker C (1997) 3D scanning PIV applied to an air flow in a motored engine using digital high-speed video. Meas Sci Technol 8:1480-1492. doi:10.1088/0957-0233/8/12/011 
Chen S, Angarita-Jaimes N, Angarita-Jaimes D, Pelc B, Greenaway AH, Towers CE, Lin D, Towers PD (2009) Wavefront sensing for three-component three-dimensional flow velocimetry in microfluidics. Exp Fluids 47:849-863. doi: $10.1007 / \mathrm{s} 00348-009-0737-\mathrm{Z}$

Choi YS, Lee SJ (2009) Three-dimensional volumetric measurement of red blood cell motion using digital holographic microscopy. Appl Opt 48:2983-2990. doi:10.1364/AO.48.002983

Choi YS, Lee SJ (2010) Hologtraphic analysis of three-dimensional inertial migration of spherical particles in micro-scale pipe flow. Microfluid Nanofluid 9:819-829. doi:10.1007/s10404-010-0601-8

Cierpka C, Segura R, Hain R, Kähler CJ (2010) A simple single camera 3C3D velocity measurement technique without errors due to depth of correlation and spatial averaging for micro fluidics. Meas Sci Technol 21:045401. doi: $10.1088 / 0957-0233 / 21 / 4 / 045401$

Cierpka C, Rossi M, Segura R, Kähler CJ (2011) On the calibration of astigmatism particle tracking velocimetry for microflows. Meas Sci Technol 22:015401. doi:10.1088/0957-0233/22/1/015401

Cierpka C, Rossi M, Segura R, Mastrangelo F, Kähler CJ (2011b) A comparative analysis of the uncertainty of astigmatism$\mu$ PTV, stereo- $\mu$ PIV, and $\mu$ PIV. Exp Fluids. doi:10.1007/s00348-011-1075-5

Coëtmellec S, Buraga-Lefebvre C, Lebrun D, Özkul C (2001) Application of in-line digital holography to multiple plane velocimetry. Meas Sci Technol 12:1392-1397. doi:10.1088/0957-0233/12/9/303

Dalgarno PA, Dalgarno HI, Putoud A, Lambert R, Paterson L, Logan DC, Towers DP, Warburton RJ, Greenaway AH (2010) Multiplane imaging and three dimensional nanoscale particle tracking in biological microscopy. Opt Express 18:877-884. doi:10.1364/OE.18.000877

Devasenathipathy S, Santiago JG, Wereley ST, Meinhart CD, Takehara K (2003) Particle imaging techniques for microfabricated fluidic systems. Exp Fluids 34:504-514. doi:10.1007/s00348-003-0588-y

Di Leonardo R, Leach J, Mushfique H, Cooper JM, Ruocco G, Padgett MJ (2006) Multipoint holographic optical velocimetry in microfluidic systems. Phys Rev Lett 96:134502. doi:10.1103/PhysRevLett.96.134502

Duncan J, Dabiri D, Hove J, Gharib M (2010) Universal outlier detection for particle image velocimetry (PIV) and particle tracking velocimetry (PTV) data. Exp Fluids 21:057002. doi:10.1088/0957-0233/21/5/057002

Elsinga GE, Scarano F, Wieneke B, van Oudheusden BW (2006) Tomographic particle tracking velocimetry. Exp Fluids 41:933-947. doi:10.1007/s00348-006-0212-z

Elsinga G, Westerweel J, Scarano F, Novara M (2011) On the velocity of ghost particles and the bias errors in tomographicPIV. Exp Fluids 50:825-838. doi:10.1007/s00348-010-0930-0

Erkan N, Shinohara K, Someya S, Okamoto K (2008) Three-component velocity measurement in microscale flows using timeresolved PIV. Meas Sci Technol 19:057003. doi:10.1088/0957-0233/19/5/057003

Fouras A, Jacono DL, Nguyen CV, Hourigan K (2009) Volumetric correlation PIV: a new technique for 3D velocity vector field measurement. Exp Fluids 47:569-577. doi:10.1007/s00348-009-0616-7

Gabor D (1948) A new microscopic principle. Nature 161:777-778. doi:10.1038/161777a0

Gazzola D, Franchi Scarselli E, Guerrieri R (2009) 3D visualization of convection patterns in lab-on-chip with open microfluidic outlet. Micro Nano 7:659-668. doi:10.1007/s10404-009-0426-5

Giardino J, Hertzberg J, Bradley E (2008) A calibration procedure for millimeter-scale stereomicroscopic particle image velocimetry. Exp Fluids 45:1037-1045. doi:10.1007/s00348-008-0525-1

Gösch M, Blom H, Holm J, Heino T, Rigler R (2000) Hydrodynamic flow profiling in microchannel structures by single molecule fluorescence correlation spectroscopy. Anal Chem 14:3260-3265. doi:10.1021/ac991448p

Grothe RL, Dabiri D (2008) An improved three-dimensional characterization of defocusing digital particle image velocimetry (DDPIV) based on a new imaging volume definition. Meas Sci Technol 19:065402. doi:10.1088/0957-0233/19/6/065402

Guerrero JA, Mendoza-Santoyo F, Moreno D, Funes-Gallanzi M, Fernandez-Orozco S (2000) Particle positioning from CCD images: experiments and comparison with the generalized LorenzMie theory. Meas Sci Technol 11:568-575. doi: $10.1088 / 0957-0233 / 11 / 5 / 318$

Guerrero-Viramontes JA, Moreno-Hernández D, Mendoza-Santoyo F, Funes-Gallanzi M (2006) 3D particle positioning from CCD images using the generalized LorenzMie and Huygens-Fresnel theory. Meas Sci Technol 17:2328-2334. doi: $10.1088 / 0957-0233 / 17 / 8 / 039$

Hagsäter SM, Westergard CH, Bruus H, Kutter JP (2008) Investigations on LED illumination for micro-PIV including a novel front-lit configuration. Exp Fluids 44:211-219. doi:10.1007/s00348-007-0394-z

Hain R, Kähler CJ (2006) Single camera volumetric velocity measurements using optical aberrations. In: 12 th international symposium on flow visualization, Göttingen, Germany

Hain R, Kähler CJ (2007) Fundamentals of multiframe particle image velocimetry (PIV). Exp Fluids 42:575-587. doi: $10.1007 / \mathrm{s} 00348-007-0266-6$

Hain R, Kähler CJ, Tropea C (2007) Comparison of CCD, CMOS and intensified cameras. Exp Fluids 42:403-411. doi: 10.1007/s00348-006-0247-1

Hain R, Kähler CJ, Radespiel R (2009) Principles of a volumetric velocity measurement technique based on optical aberrations. In: Notes on numerical fluid mechanics and multidisciplinary design, vol 106. Springer, Berlin, pp 1-10. doi: 10.1007/978-3-642-01106-1_1

Hinsch KD (2002) Holographic particle image velocimetry. Meas Sci Technol 13:R61-R72. doi:10.1088/0957-0233/13/7/201

Hiraoka Y, Sedat JW, Agard DA (1990) Determination of three-dimensional imaging properties of a light microscope system. Partial confocal behavior in epifluorescence microscopy. Biophys J 57:325-333. doi:10.1016/S0006-3495(90)82534-0

Hoffmann M, Schlüter M, Räbiger N (2007) Untersuchungen der Mischvorgänge in Mikroreaktoren durch Anwednung der Micro-LIF und Micro-PIV. Chem Ing Tech 79:1067-1075. doi:10.1002/cite200700064

Holtzer L, Meckel T, Schmidt T (2007) Nanometric three-dimensional tracking of individual quantum dots in cells. Appl Phys Lett 90:053902. doi:10.1063/1.2437066

Hsu W, Lee CS, Chen PJ, Chen NT, Chen FZ, Yu ZR, Kuo CH, Hwang CH (2009) Development of the fast astigmatism autofocus microscope system. Meas Sci Technol 20:045902. doi:10.1088/0957-0233/20/4/045902 
Huang B, Wang W, Bates M, Zhuang X (2008) Three-dimensional super-resolution imaging by stochastic optical reconstruction microscopy. Science 319:810-813. doi:10.1126/science.1153529

Ichiyanag M, Sato Y, Hishida K (2007) Optically sliced measurement of velocity and pH distribution in microchannel. Exp Fluids 43:425-435. doi:10.1007/s00348-007-0326-y

Ismagilov RF, Stroock AD, Kenis PJA, Whitesides G, Stone HA (2000) Experimental and theoretical scaling laws for transverse diffusive broadening in two-phase laminar flows in microchannels. Appl Phys Lett 76(17):2376-2378. doi: $10.1063 / 1.126351$

Kähler CJ (2004) The significance of coherent flow structures for the turbulent mixing in wall-bounded flows. PhD thesis, Georg-August-Universität zu Göttingen. http://webdoc.sub.gwdg.de/diss/2004/kaehler/kaehler.pdf

Kähler CJ, Scharnowski S (2011) On the resolution limit of digital particle image velocimetry. In: 9th international symposium on PIV, Kobe, Japan

Kähler CJ, Adrian RJ, Willert C (1998) Turbulent boundary layer investigations with conventional and stereoscopic particle image velocimetry. In: 9th international symposium on applications of laser techniques to fluid mechanics, Lisbon, Portugal

Kähler CJ, Scholz U, Ortmanns J (2006) Wall-shear-stress and near-wall turbulence measurements up to single pixel resolution by means of long-distance micro-PIV. Exp Fluids 41:327-341. doi:10.1007/s00348-006-0167-0

Kajitani L, Dabiri D (2005) A full three-dimensional characterization of defocusing digital particle image velocimetry. Meas Sci Technol 16:790-804. doi:10.1088/0957-0233/16/3/022

Kajitani L, Dabiri D (2008) A full three-dimensional characterization of defocusing digital particle image velocimetry. Meas Sci Technol 19:049801. doi:10.1088/0957-0233/19/4/049801

Kao HP, Verkman AS (1994) Tracking of single fluorescent particles in three dimensions: use of cylindrical optics to encode particle position. Biophys J 67:1291-1300. doi:10.1016/S0006-3495(94)80601-0

Katz J, Sheng J (2010) Applications of holography in fluid mechanics and particle dynamics. Ann Rev Fluid Mech 42:531-555. doi:10.1146/annurev-fluid-121108-145508

Keane RD, Adrian RJ, Zhang Y (1995) Super-resolution particle imaging velocimetry. Meas Sci Technol 6:754-768. doi: $10.1088 / 0957-0233 / 6 / 6 / 013$

Kim H, Groe S, Elsinga G, Westerweel J (2011) Full 3D-3C velocity measurement inside a liquid immersion droplet. Exp Fluids. doi:10.1007/s00348-011-1053-y

Kinoshita H, Kaneda S, Fujii T, Oshima M (2007) Three-dimensional measurement and visualization of internal flow of a moving droplet using confocal micro-PTV. Lab Chip 7:338-346. doi:10.1039/lb617391h

Klank H, Goranovic G, Kutter JP, Gjelstrup H, Michelsen J, Westergaard CH (2002) PIV measurements in a microfluidic 3D-sheathing structure with three-dimensional flow behaviour. J Micromech Microeng 12:862-869. doi: 10.1088/0960-1317/12/6/318

Klasen LG, Leder A, Brede M (2005) Entwicklung und Aufbau eines Stereo-Mikro-PIV Systems zur instantanen Strömungsfeldmessung. In: 13. Fachtagung der GALA e.V., Cottbus, Germany

Klein SA, Posner JD (2010) Improvement in two-frame correlations by confocal microscopy for temporally resolved micro particle imaging velocimetry. Meas Sci Technol 21:105409. doi:10.1088/0957-0233/21/10/105409

Kloosterman A, Poelma C, Westerweel J (2010) Flow rate estimation in large depth-of-field micro-PIV. Exp Fluids. doi: 10.1007/s00348-010-1015-9

Koutsiaris AG, Mathioulakis DS, Tsangaris S (1999) Microscope PIV for velocity-field measurement of particle suspensions flowing inside glass capillaries. Meas Sci Technol 10:1037-1046. doi:10.1088/0957-0233/10/11/311

Kumar A, Cierpka C, Williams SJ, Kähler CJ, Wereley ST (2011) 3D3C velocimetry measurements of an electrothermal microvortex using wavefront deformation PTV and a single camera. Micro Nano 10:355-365. doi: 10.1007/s10404-010-0674-4

Lawson NJ, Wu J (1997) Three-dimensional particle image velocimetry: experimental error analysis of a digital angular stereoscopic system. Meas Sci Technol 8:1455-1464. doi:10.1088/0957-0233/8/12/009

Lee SJ, Kim S (2007) Measurement of 3D laminar flow inside a micro tube using micro digital holographic particle tracking velocimetry. J Micromech Microeng 17:2157-2162. doi:10.1088/0960-1317/17/10/030

Lee SJ, Kim S (2009) Advanced particle-based velocimetry techniques for microscale flows. Microfluid Nanofluid 6:577-588. doi:10.1007/s10404-009-0409-6

Lee SJ, Kim S (2009) Measurement of Dean flow in a curved micro-tube using micro digital holographic particle tracking velocimetry. Exp Fluids 46:255-264. doi:10.1007/s00348-008-0555-8

Lee SJ, Seo KW, Choi SY, Sohn MY (2011) Three-dimensional motion measurements of free-swimming microorganisms using digital holographic microscopy. Meas Sci Technol 22:064004. doi:10.1088/0957-0233/22/6/064004

Liebling M, Forouhar AS, Wolleschensky R, Zimmermann B, Ankerhold R, Fraser SE, Gharib M, Dickinson ME (2006) Rapid three-dimensional imaging and analysis of the beating embryonic heart reveals functional changes during development. Dev Dyn 235:2940-2948. doi:10.1002/dvdy.20926

Lima R, Wada S, Tsubota K, Yamaguchi T (2006) Confocal micro-PIV measurements of three-dimensional profiles of cell suspension flow in a square microchannel. Meas Sci Technol 17:797-808. doi:10.1088/0957-0233/17/4/026

Lima R, Wada S, Takeda M, Tsubota K, Yamaguchi T (2007) In vitro confocal micro-PIV measurements of blood flow in a square microchannel: the effect of the haematocrit on instantaneous velocity profiles. J Biomech 40:2752-2757. doi: 10.1016/j.biomech.2007.01.012

Lin D, Angarita-Jaimes NC, Chen S, Greenaway AH, Towers CE, Towers DP (2008) Three-dimensional particle imaging by defocusing method with an annular aperture. Opt Lett 33:905-907. doi:10.1364/OL.33.000905

Lindken R, Westerweel J, Wieneke B (2006a) 3D micro-scale velocimetry methods: a comparison between 3D- $\mu$ PTV, stereoscopic $\mu$ PIV and tomographic $\mu$ PIV. In: 13th international symposium on applications of laser techniques to fluid mechanics, Lisbon, Portugal

Lindken R, Westerweel J, Wieneke B (2006) Stereoscopic micro particle image velocimetry. Exp Fluids 41:161-171. doi: 10.1007/s00348-006-0154-5 
Lindken R, Rossi M, Grosse S, Westerweel J (2009) Micro-particle image velocimetry ( $\mu$ PIV): recent developments, applications, and guidelines. Lab Chip 9:2551-2567. doi:10.1039/b906558j

Lu J, Pereira F, Fraser SE, Gharib M (2008) Three-dimensional real-time imaging of cardiac cell motions in living embryos. J Biomed Opt 13:014006. doi:10.1117/1.2830824

Luo R, Sun YF (2011) Pattern matching for three-dimensional tracking of sub-micron fluorescent paerticles. Meas Sci Technol 22:045402. doi:10.1088/0957-0233/22/4/045402

Luo R, Yang XY, Peng XF, Sun YF (2006) Three-dimensional tracking of fluorescent particles applied to micro-fluidic measurements. J Micromech Microeng 16:1689-1699. doi:10.1088/0960-1317/16/8/034

Maas HG, Putze T, Westfeld P (2009) Recent developments in 3D-PTV and Tomo-PIV. Notes Numer Fluid Mech Multidisc Desgn 106:53-62. doi:10.1007/978-3-642-01106-16

Malek M, Allano D, Coëtmellec S, Özkul C, Lebrun D (2004) Digital in-line holography for three-dimensional twocomponents particle tracking velocimetry. Meas Sci Technol 15(4):699-705. doi:10.1088/0957-0233/15/4/012

Malkiel E, Sheng J, Katz J, Strickler RJ (2003) The three-dimensional flow field generated by a feeding calanoid copepod measured using digital holography. J Exp Biol 206:3657-3666. doi:10.1242/jeb.00586

Meinhart CD, Wereley ST (2003) The theory of diffraction-limited resolution in micro particle image velocimetry. Meas Sci Technol 14:1047-1053. doi:10.1088/0957-0233/14/7/320

Meinhart CD, Wereley ST, Santiago JG (1999) PIV measurements of a micro channel flow. Exp Fluids 27:414-419. doi: $10.1007 / \mathrm{s} 003480050366$

Meinhart CD, Wereley ST, Santiago JG (2000) A PIV algorithm for estimating time-averaged velocity fields. J Fluids Eng 122:285-289. doi: $10.1115 / 1.483256$

Meng H, Pan G, Pu Y, Woodward SH (2004) Holographic particle image velocimetry: from film to digital recording. Meas Sci Technol 15:673-685. doi:10.1088/0957-0233/15/4/009

Min YU, Kim KC (2011) Hybrid micro-/nano-particle image velocimetry for 3D3C multi-scale velocity field measurement in microfluidics. Meas Sci Technol 22:064001. doi:10.1088/0957-0233/22/6/064001

Minsky M (1961) Microscopy apparatus. US Patent 3,013,467

Mlodzianoski MJ, Juette MF, Beane GL, Bewersdorf J (2009) Experimental characterization of 3D localization techniques for particle-tracking and super-resolution microscopy. Opt Exp 17:8264-8277. doi:10.1364/OE.17.008264

Moreno-Hernandez D, Bueno-ía JA, Guerrero-Viramontes JA, Mendoza-Santoyo F (2011) 3D particle positioning by using the Fraunhofer criterion. Opt Laser Eng. doi:10.1016/j.optlaseng.2011.01.019

Murata S, Kawamura M (1999) Particle depth measurement based on depth-from-defocus. Opt Laser Technol 31:95-102. doi: 10.1002/aic.10165

Nasarek R (2010) Temperature field measurements with high spatial and temporal resolution using liquid crystal thermography and laser induced fluorescence. $\mathrm{PhD}$ thesis, Technische Universität Darmstadt, Germany

Nguyen C, Fouras A, Carberry J (2010) Improvement of measurement accuracy in micro PIV by image overlapping. Exp Fluids 49:701-712. doi:10.1007/s00348-010-0837-9

Nguyen D, Honnery D, Soria J (2010) Measuring evaporation of micro-fuel droplets using magnified DIH and DPIV. Exp Fluids 50:1-11. doi:10.1007/s00348-010-0962-5

Olsen MG, Adrian RJ (2000) Out-of-focus effects on particle image visibility and correlation in microscopic particle image velocimetry. Exp Fluids 29:S166-S174. doi:10.1007/s003480070018

Ooms TA, Koek W, Braat J, Westerweel J (2006) Optimizing Fourier filtering for digital holographic particle image velocimetry. Meas Sci Technol 17:304-312. doi:10.1088/0957-0233/17/2/011

Ooms TA, Lindken R, Westerweel J (2009) Digital holographic microscopy applied to measurement of a flow in a T-shaped micromixer. Exp Fluids 47:941-955. doi:10.1007/s00348-009-0683-9

Ovryn B, Hovenac EA (1993) Coherent forward scattering particle-image velocimetry: application of Poisson's spot for velocity measurements in fluids. In: Optical diagnostics in fluid and thermal flow, San Diego, CA, USA, pp 338-348. doi: $10.1117 / 12.163718$

Padilla Sosa P, Moreno D, Guerrero JA, Funes-Gallanzi M (2002) Low-magnification particle positioning for 3D velocimetry applications. Opt Laser Technol 34:59-68. doi:10.1016/S0030-3992(01)00096-2

Park JS, Khim KD (2006) Three-dimensional micro-PTV using deconvolution microscopy. Exp Fluids 40:491-499. doi: 10.1007/s00348-005-0090-9

Park JS, Choi CK, Kihm K (2004) Optically sliced micro-PIV using confocal laser scanning microscopy CLSM. Exp Fluids 37:105-119. doi:10.1007/s00348-004-0790-6

Park JS, Khim KD, Lee SJ (2010) Nonintrusive measurements of mixture concentration fields by analysing diffraction image patterns (point spread function) of nanoparticles. Exp Fluids 49:183-191. doi:10.1007/s00348-010-0831-2

Pereira F, Gharib M (2002) Defocusing digital particle image velocimetry and three-dimensional characterization of two phase flows. Meas Sci Technol 13:683-694. doi:10.1088/0957-0233/13/5/305

Pereira F, Gharib M, Dabiri D, Modarress D (2000) Defocusing digital particle image velocimetry: a 3-component 3-dimensional DPIV measurement technique. Application to bubbly flows. Exp Fluids 29:S78-S84. doi: $10.1007 / \mathrm{s} 003480070010$

Pereira F, Lu J, Castaño Graff E, Gharib M (2007) Microscale 3D flow mapping with $\mu$ DDPIV. Exp Fluids 42:589-599. doi: $10.1007 / \mathrm{s} 00348-007-0267-5$

Peterson SD, Chuang HS, Wereley ST (2008) Three-dimensional particle tracking using micro-particle image velocimetry hardware. Meas Sci Technol 19:115-406. doi:10.1088/0957-0233/19/11/115406

Poelma C, Westerweel J (2010) Generalized displacement estimation for averages of non-stationary flows. Exp Fluids. doi: $10.1007 / \mathrm{s} 00348-010-1002-1$

Prasad AK (2000) Stereoscopic particle image velocimetry. Exp Fluids 29:103-116. doi:10.1007/s003480000143

Racca R, Dewey J (1988) A method for automatic particle tracking in a three-dimensional flow field. Exp Fluids 6:25-32. doi: $10.1007 / \mathrm{BF} 0022613$ 
Raffel M, Westerweel J, Willert C, Gharib M, Kompenhans J (1996) Analytical and experimental investigations of dual-plane particle image velocimetry. Opt Eng 35:2067-2074. doi:10.1117/1.600695

Raffel M, Willert C, Wereley ST, Kompenhans J (2007) Particle image velocimetry. A practical guide. Springer, Berlin

Ragan T, Huang H, So P, Gratton E (2006) 3D particle tracking on a two-photon microscope. J Fluorescence 16:325-336. doi: 10.1007/s10895-005-0040-1

Rohály J, Lammerding J, Frigerio F, Hart DP (2001) Monocular 3-D active $\mu$-PTV. In: 4th international symposium on PIV, Göttingen, Germany

Rossi M, Lindken R, Hierck BP, Westerweel J (2009) Tapered microfluidic chip for the study of biochemical and mechanical response of endothelial cells to shear flow at subcellular level. Lab Chip 9:1403-1411. doi:10.1039/B822270N

Rossi M, Lindken R, Westerweel J (2010) Optimization of multiplane $\mu$ PIV for wall shear stress and wall topography characterization. Exp Fluids 48:211-223. doi:10.1007/s00348-009-0725-3

Rossi M, Cierpka C, Segura R, Kähler CJ (2011) Volumetric reconstruction of the 3D boundary of stream tubes with general topology using tracer particles. Meas Sci Technol 22:105-405. doi:10.1088/0957-0233/22/10/105405

Rossi M, Segura R, Cierpka C, Kähler CJ (2011b) On the effect of particle image intensity and image preprocessing on depth of correlation in micro-PIV measurements. Exp Fluids. doi:10.1007/s00348-011-1194-Z

Samarage CR, Carberry J, Hourigan K, Fouras A (2011) Optimization of temporal averaging processes in PIV. Exp Fluids. doi: 10.1007/s00348-011-1080-8

Santiago JG, Wereley ST, Meinhart CD, Beebe DJ, Adrian RJ (1998) A particle image velocimetry system for microfluidics. Exp Fluids 25:316-319. doi:10.1007/s003480050235

Satake S, Kunugi T, Sato K, Ito T (2004) Digital holographic particle tracking velocimetry for 3-D transient flow around an obstacle in a narrow channel. Opt Rev 11:162-164. doi:10.1007/s10-043-0042-4

Satake S, Kunugi T, Sato K, Ito T, Taniguchi J (2005) Three-dimensional flow tracking in a micro channel with high time resolution using micro digital-holographic particle-tracking velocimetry. Opt Rev 12:442-444. doi: 10.1007/s10043-005-0442-y

Satake S, Kunugi T, Sato K, Ito T, Kanamori H, Taniguchi J (2006) Measurements of 3D flow in a micro-pipe via micro digital holographic particle tracking velocimetry. Meas Sci Technol 17:1647-1651. doi:10.1088/0957-0233/17/7/002

Sato Y, Inabe S, Hishida K, Maeda M (2003) Spatially averaged time-resolved particle-tracking velocimetry in microspace considering Brownian motion of submicron fluorescent particles. Exp Fluids 35:167-177. doi:10.1007/s00348-003-0643-8

Scharnowski S, Hain R, Kähler CJ (2011) Reynolds stress estimation up to single-pixel resolution using PIV-measurements. Exp Fluids. doi:10.1007/s00348-011-1184-1

Schnars U, Jüptner PO (1994) Direct recording of holograms by a CCD target and numerical reconstruction. Appl Opt 33:179-181. doi:10.1364/AO.33.000179

Schnars U, Jüptner PO (2002) Digital recording and numerical reconstruction of holograms. Meas Sci Technol 13:R85-R101. doi:10.1088/0957-0233/13/9/201

Shinohara K, Sugii Y, Jeong JH, Okamoto K (2005) Development of a three-dimensional scanning microparticle image velocimetry system using a piezo actuator. Rev Sci Instrum 76:106-109. doi:10.1063/1.2114889

Sinton D (2004) Microscale flow visualization. Micro Nano 1:2-21. doi:10.1007/s10404-004-0009-4

Soria J, Atkinson C (2008) Towards 3C-3D digital holographic fluid velocity vector field measurements: tomographic digital holographic PIV (Tomo-HPIV). Meas Sci Technol 19:074002. doi:10.1088/0957-0233/19/7/074002

Speidel M, Jonáš EL, Florin A (2000) Three-dimensional tracking of fluorescent nanoparticles with subnanometer precision by use of off-focus imaging. Opt Lett 28:69-71. doi:10.1364/OL.28.000069

Squires TM, Quake SR (2005) Microfluidics: fluid physics at the nanoliter scale. Rev Mod Phys 77:977-1026. doi: 10.1103/RevModPhys.77.977

Stanislas M, Okamoto K, Kähler CJ (2003) Main results of the first international PIV challenge. Meas Sci Technol 14:R63. doi: $10.1088 / 0957-0233 / 14 / 10 / 201$

Stolz W, Köhler J (1994) In-plane determination of 3D-velocity vectors using particle tracking anemometry (PTA). Exp Fluids 17:105-109. doi:10.1007/BF02412811

Sugii Y, Okamoto K (2003) Quantitative visualization of micro-tube flow using micro-PIV. J Vis 7:9-16. doi: $10.1007 / \mathrm{BF} 03181480$

Sun Y, Duthaler S, Nelson BJ (2004) Autofocusing in computer microscopy: selecting the optimal focus algorithm. Microsc Res Tech 65:139-149. doi:10.1002/jemt.20118

Tien WH, Kartes P, Yamasaki T, Dabiri D (2008) A color-coded backligthed defocusing digital particle image velocimetry system. Exp Fluids 44:1015-1026. doi:10.1007/s00348-007-0457-1

Toprak E, Balci H, Blehm BH, Selvin PR (2007) Three-dimensional particle tracking via bifocal imaging. Nano Lett 7:2043-2045. doi:10.1021/nl0709120

Towers CE, Towers DP, Campbell HI, Zhang S, Greenaway AH (2006) Three-dimensional particle imaging by wavefront sensing. Opt Lett 31:1220-1222. doi:10.1364/OL.31.001220

Tropea C, Yarin AL, Foss JFE (2007) Springer handbook of experimental fluid mechanics. Springer. ISBN:978-3-540-25141-5

van Hinsberg NP, Roisman IV, Tropea C (2008) Three-dimensional, three-component particle imageing using two optical aberrations and a single camera. In: 14th international symposium on applications of laser techniques to fluid mechanics, Lisbon, Portugal

Wereley ST, Meinhart CD (2010) Recent advances in micro particle image velocimetry. Ann Rev Fluid Mech 42:557-576. doi: 10.1146/annurev-fluid-121108-145427

Westerweel J (1997) Fundamentals of digital particle image velocimetry. Meas Sci Technol 8:1379-1392. doi: 10.1088/0957-0233/8/12/002

Westerweel J, Scarano F (2005) Universal outlier detection for PIV data. Exp Fluids 39:1096-1100. doi: $10.1007 / \mathrm{s} 00348-005-0016-6$

Westerweel J, Geelhoed PF, Lindken R (2004) Single-pixel resolution ensemble correlation for micro-PIV applications. Exp Fluids 37:375-384. doi:10.1007/s00348-004-0826-y 
Wieneke B (2005) Stereo-PIV using self-calibration on particle images. Exp Fluids 39:267-280. doi: $10.1007 / \mathrm{s} 00348-005-0962-\mathrm{z}$

Willert C, Gharib M (1992) Three-dimensional particle imaging with a single camera. Exp Fluids 12:353-358. doi: 10.1007/BF00193880

Williams SJ, Park C, Wereley ST (2010) Advances and applications on microfluidic velocimetry techniques. Micro Nano 8:709-726. doi:10.1007/s10404-010-0588-1

Worth N, Nickels T (2008) Acceleration of Tomo-PIV by estimating the initial volume intensity distribution. Exp Fluids 45:847-856. doi:10.1007/s00348-008-0504-6

Wu M, Roberts JW, Buckley M (2005) Three-dimensional fluorescent particle tracking at micron-scale using a single camera. Exp Fluids 38:461-465. doi:10.1007/s00348-004-0925-9

Yamamoto Y, Uemura T (2008) 3D particle measurements by single beam two-views magnified digital in-line holography. Exp Fluids 45:813-821. doi:10.1007/s00348-008-0501-9

Yang CT, Chuang HS (2005) Measurement of a microchamber flow by using a hybrid multiplexing holographic velocimetry. Exp Fluids 39:385-396. doi:10.1007/s00348-005-1022-4

Yoon SY, Kim KC (2006) 3D particle and 3D velocity field measurement in a microvolume via the defocusing concept. Meas Sci Technol 17:2897-2905. doi:10.1088/0957-0233/17/11/006

Yoon SY, Yang S (2010) Microfluidic device for refractive index measurement of fluid sample. Proc Eng 5:1340-1343. doi: 10.1016/j.proeng.2010.09.362

Yoon SY, Khim KD, Kim KC (2011) Correlation of fluid refractive index with calibration coefficient for micro-defocusing digital particle image velocimetry. Meas Sci Technol 22:037001. doi:10.1088/0957-0233/22/3/037001

Yoshida H (2005) The wide variety of possible applications of micro-thermofluid control. Microfluid Nanofluid 1:289-300 doi:10.1007/s10404-004-0014-7

Yu CH, Yoon JH, Kim HB (2009) Development and validation of stereoscopic micro-PTV using match probability. J Mech Sci Tech 23:1-7. doi:10.1007/s12206-008-1209-8 